\title{
Long Nonlinear Internal Waves
}

\author{
Karl R. Helfrich ${ }^{1}$ and W. Kendall Melville ${ }^{2}$ \\ ${ }^{1}$ Department of Physical Oceanography, Woods Hole Oceanographic Institution, Woods Hole, \\ Massachusetts 02543; email: khelfrich@whoi.edu \\ ${ }^{2}$ Scripps Institution of Oceanography, University of California, San Diego, La Jolla, California 92093-0213; \\ email: kmelville@ucsd.edu
}

Annu. Rev. Fluid Mech.
2006. 38:395-425

The Annual Review of Fluid Mechanics is online at fluid.annualreviews.org

doi: 10.1146/annurev.fluid. 38.050304 .092129

Copyright (c) 2006 by Annual Reviews. All rights reserved

0066-4189/06/0115$0395 \$ 20.00$

\section{Key Words}

solitary waves, nonlinear waves, stratified flow, physical oceanography

\begin{abstract}
Over the past four decades, the combination of in situ and remote sensing observations has demonstrated that long nonlinear internal solitary-like waves are ubiquitous features of coastal oceans. The following provides an overview of the properties of steady internal solitary waves and the transient processes of wave generation and evolution, primarily from the point of view of weakly nonlinear theory, of which the Korteweg-de Vries equation is the most frequently used example. However, the oceanographically important processes of wave instability and breaking, generally inaccessible with these models, are also discussed. Furthermore, observations often show strongly nonlinear waves whose properties can only be explained with fully nonlinear models.
\end{abstract}




\section{INTRODUCTION}

Much of the modern interest in large internal waves in oceanography began in the 1960 s to 1970 s with an interesting confluence of events in ocean instrumentation, applied mathematics, and remote sensing. The development of fast internally recording vertical arrays ("chains") of thermistors in the 1960s led to observations of large internal waves in the coastal oceans and marginal seas. Among the most dramatic of the early measurements were those of Perry \& Schimke (1965) in the Andaman Sea. They found groups of internal waves up to $80 \mathrm{~m}$ high and $2000 \mathrm{~m}$ long on the main thermocline at $500 \mathrm{~m}$ in water $1500-\mathrm{m}$ deep. Osborne \& Burch (1980) subsequently showed that the waves were generated by tidal flows through the channels in the Andaman and Nicobar island chains and propagated toward the Sumatra coastline some hundreds of kilometers away. Among the other early ocean observations were those of Ziegenbein $(1969,1970)$ in the Strait of Gibraltar and Halpern (1971) in Massachusetts Bay (also see Haury et al. 1979). About the same time, Thorpe (1971) and Hunkins \& Fliegel (1973) were making similar observations in Loch Ness and Seneca Lake, New York, respectively. Zeigenbein's observations were particularly notable for the clear evidence of a "singular" (solitary) wave.

What became apparent in the early observations was that these were not linear dispersive waves. The heights of the waves compared to the appropriate vertical scales of the stratification were too large for them to be linear. The fact that they remained coherent and of finite amplitude for long distances implied that dispersion was not dominant. The canonical equation for the evolution of long free waves with competing nonlinear and dispersive effects is the Korteweg-de Vries (KdV) equation, and the early field observers were aware of the then recent discovery by Gardner et al. (1967) of exact asymptotic solutions of the $\mathrm{KdV}$ equation corresponding to rank-ordered solitary waves (Hunkins \& Fliegel 1973).

The third component was remote sensing. Ziegenbein (1969) demonstrated that the presence of internal waves could be inferred from scattering of marine radar from short surface waves. Apel et al. (1975) showed evidence of large internal wave groups in the New York Bight and the southwest coast of Africa, but it was the launch of SEASAT in 1978 and the synthetic aperture radar (SAR) images of the coastal oceans that demonstrated that packets of shoreward-propagating internal waves, separated by tidal periods, were a ubiquitous feature of the coastal oceans. Many examples of these early images can be found in Fu \& Holt (1982), who commented that images of internal wave signatures were a major component of the SEASAT SAR observations. An extensive collection of images can be found at http://www.internalwaveatlas.com/.

The SAR image in Figure 1 from Fu \& Holt (1982) is illustrative. The image of the Gulf of California shows the surface signature of at least eight wave packets generated by stratified tidal flow over the steep topography in the channels between Baja California and nearby islands. The curved wave fronts and variation in wavelength within a group indicate that the packets are propagating toward shallower water to the north-northeast. As the waves propagate they evolve due to the changing topography, currents, and stratification, and dissipate. Dissipation by breaking may result in significant vertical mixing that is important for a number of coastal processes. 

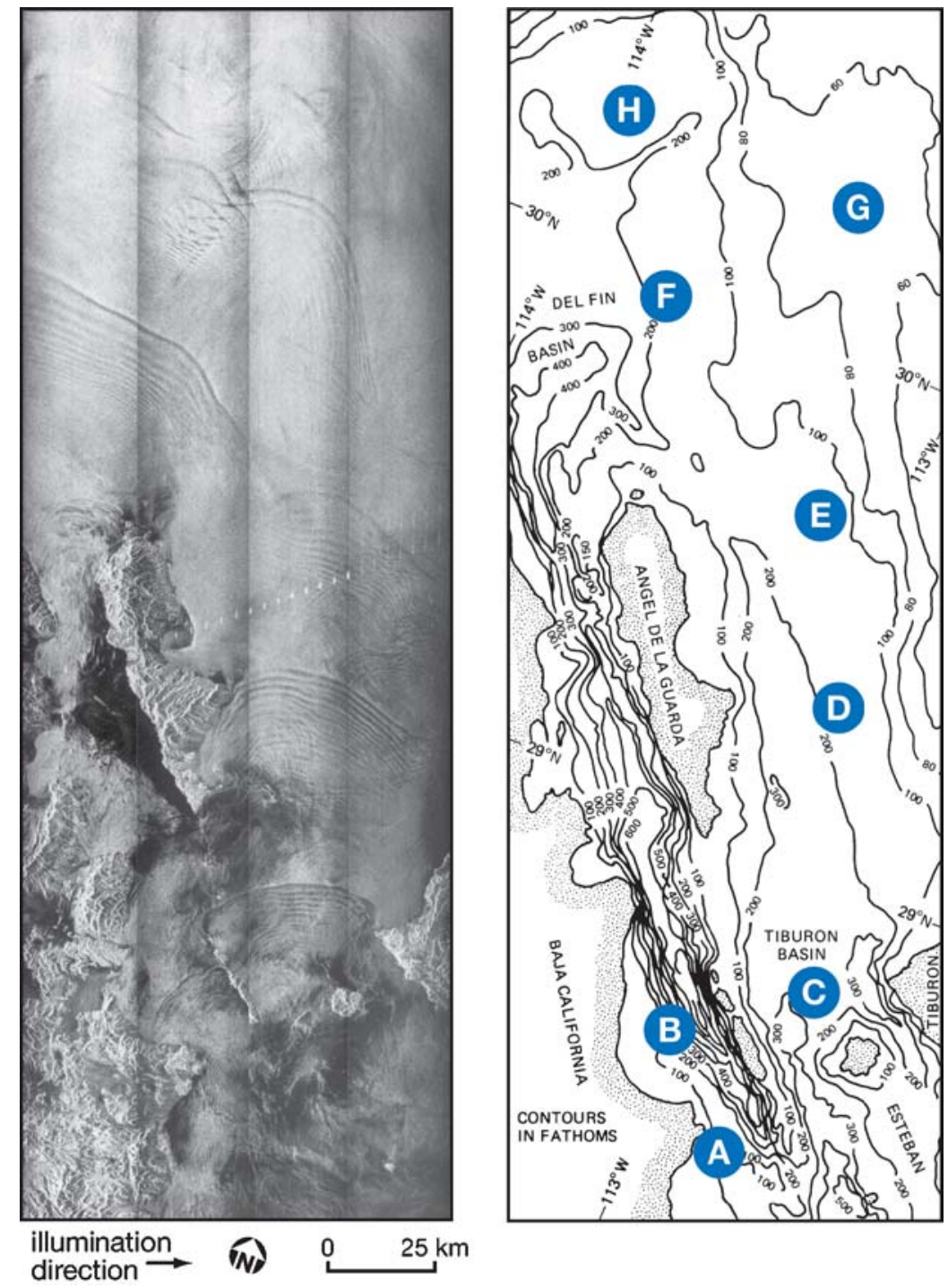

Another interesting aspect of the wave evolution is the strong interactions between waves in groups $\mathrm{G}$ and $\mathrm{H}$ evident in the complex wave patterns.

Two in situ observations from thermistor arrays are given in Figure 2. Both are notable for the strong nonlinearity of the waves and also show the common situation of waves "pointing" into the deeper layer. Figure $2 \boldsymbol{a}$ from Stanton \& Ostrovsky (1998) shows the leading portion of a packet of waves propagating toward the coast on the Oregon shelf. Wave amplitudes are $20-25 \mathrm{~m}$ on a upper layer of just $7-\mathrm{m}$ depth. Here and throughout the review wave amplitude is defined as the maximum displacement
Figure 1

Synthetic aperature radar image of a northern portion of the Gulf of California from Fu \& Holt (1982). The internal waves are visible as alternating light and dark bands. The right panel shows the bathymetry of the region and the letters indicate the locations of eight separate wave groups visible in the SAR image. 
from the undisturbed flow. The ratio of the amplitude to the upper-layer depth is approximately 3 , well beyond the assumptions of a weakly nonlinear theory. The isolated wave observed in the South China Sea [Figure $2 \boldsymbol{b}$ from Duda et al. (2004)] illustrates the tendency for waves with amplitudes that are a significant fraction of the total depth to broaden and develop a flat crest. The amplitude is about $150 \mathrm{~m}$ in water of 340-m total depth with a background upper-layer depth of about $40 \mathrm{~m}$. The heavy dashed line is the profile for a KdV solitary wave Duda et al. (2004) calculated for the observed conditions, and illustrates that the observed wave is qualitatively different.

The focus of this review is oceanic waves; however, similar phenomena occur in the lower atmosphere. One of the most striking examples is the Morning Glory bore of the Gulf of Carpenteria in northeast Australia (Christie 1992). Another compelling atmospheric observation is shown in Figure 3 (from Li et al. 2004). The visible image from the MODIS satellite is of St. Lawrence Island in the Bering Strait. The packet of 7-10 waves just north of the island is visible because of cloud formation due to the wave-induced uplift. Unlike the typical oceanic case, these waves were not generated by tidal flow, but by resonant forcing of the steady southward lower atmospheric flow by the island topography. Nonlinearity allows the waves to propagate upstream into the flow.

Topics related to the material covered in this review have been of interest in geophysical fluid dynamics, oceanography, and meteorology for a generation of researchers. During that time, excellent reviews covering some aspects of long nonlinear internal waves, or closely related topics, have been published. The reader is referred to Miles (1980) for a review on solitary waves, including internal solitary waves. Ostrovsky \& Stepanyants (1989) reviewed field observations and theories of nonlinear internal waves, and internal solitary waves in particular. Grimshaw (1997) and Grimshaw et al. (1998) covered internal solitary waves, and long nonlinear waves in rotating systems, respectively. Finally, the review by Akylas (1994) of three-dimensional long nonlinear waves is also relevant.

\section{SOLITARY WAVE MODELS}

Despite the fact that the oceanic observations show mode-one internal waves that are often highly nonlinear, weakly nonlinear $\mathrm{KdV}$-type theories have played the primary role in elucidating the essential features of the observations, if not always the precise quantitative details. They have the advantage of permitting modeling of unsteady wave evolution under various conditions with a reduced wave equation, or equations. Fully nonlinear theories for solitary waves are available to extend the solitary wave solutions to large amplitudes. However, these models are generally restricted to steady solitary waves or to computationally expensive time-dependent solutions of the Euler or Navier-Stokes equations. Wave evolution and dissipation can be studied, but the ease of generalization is often lost.

To set the stage for the discussion of wave evolution (below), important results for steady internal solitary waves from both weakly and fully nonlinear wave models and laboratory experiments are described. 
a

Temperature $\left({ }^{\circ} \mathrm{C}\right)$

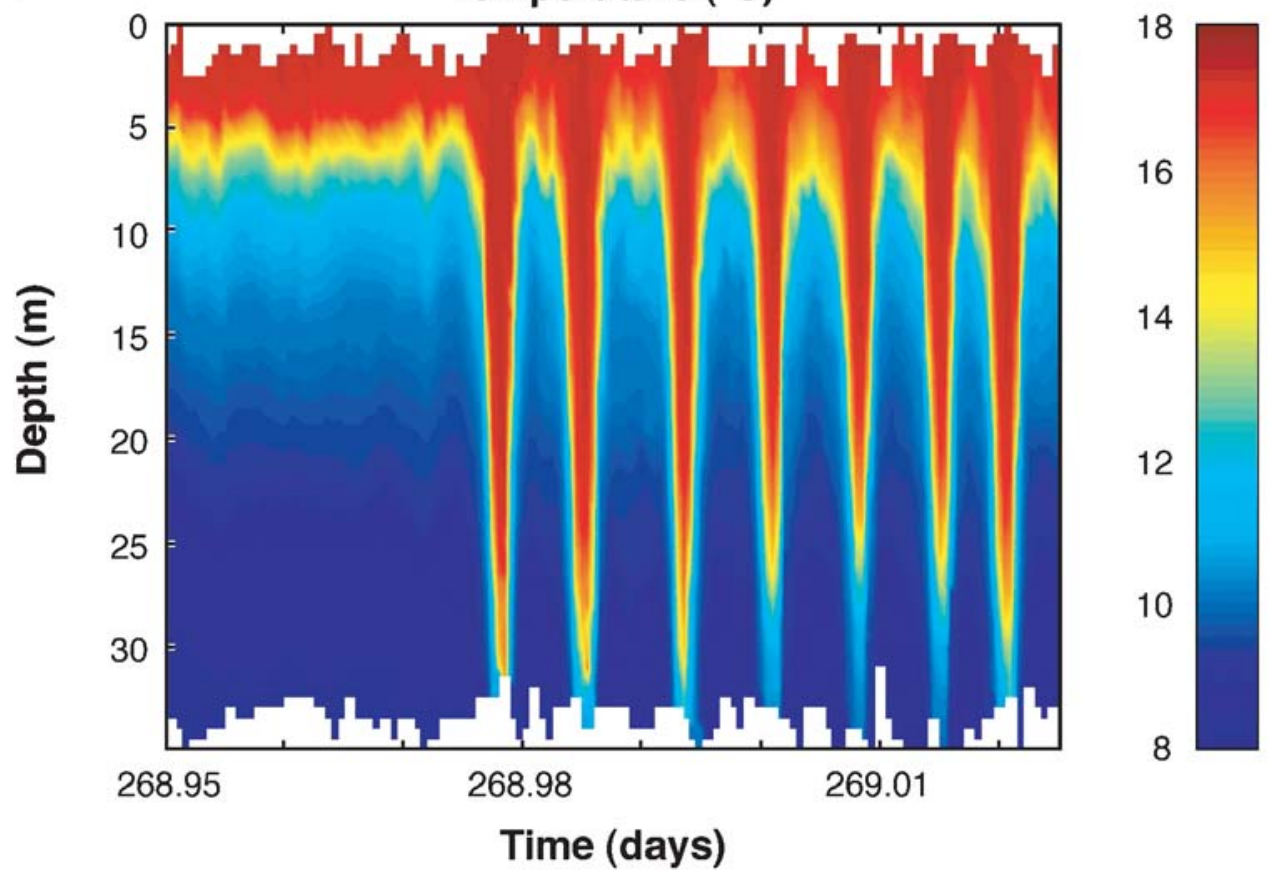

b

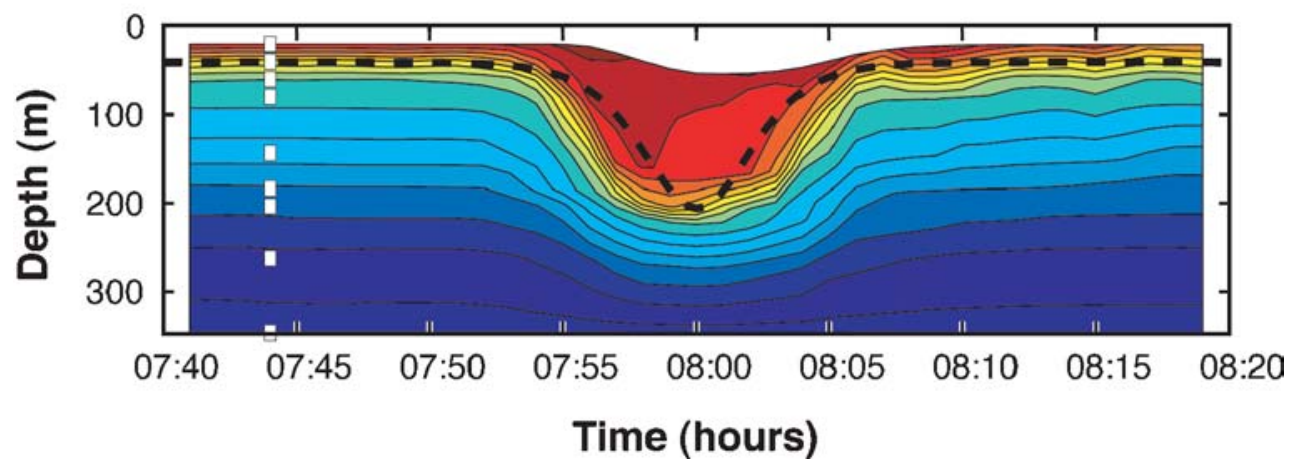

Figure 2

Large-amplitude internal waves observed with fixed thermistor arrays. (a) The leading portion of a wave packet observed in about $147 \mathrm{~m}$ of water and propagating toward the Oregon coast (Stanton \& Ostrovsky 1998). The colors indicate temperatures as indicated in the color bar. (b) A single large wave in $340 \mathrm{~m}$ of water in the northeast South China Sea (from Duda et al. 2004). The temperature is contoured in intervals of $1^{\circ} \mathrm{C}$, and the white squares indicate the thermistor locations. The heavy dashed line is the profile of a KdV solitary wave calculated using the background stratification. 
Figure 3

MODIS image of the Bering Sea showing a packet of about seven atmospheric solitary-like waves propagating north from St. Lawrence Island (from Li et al. 2004). The waves are generated by the near critical southward lower atmosphere flow over the island topography.

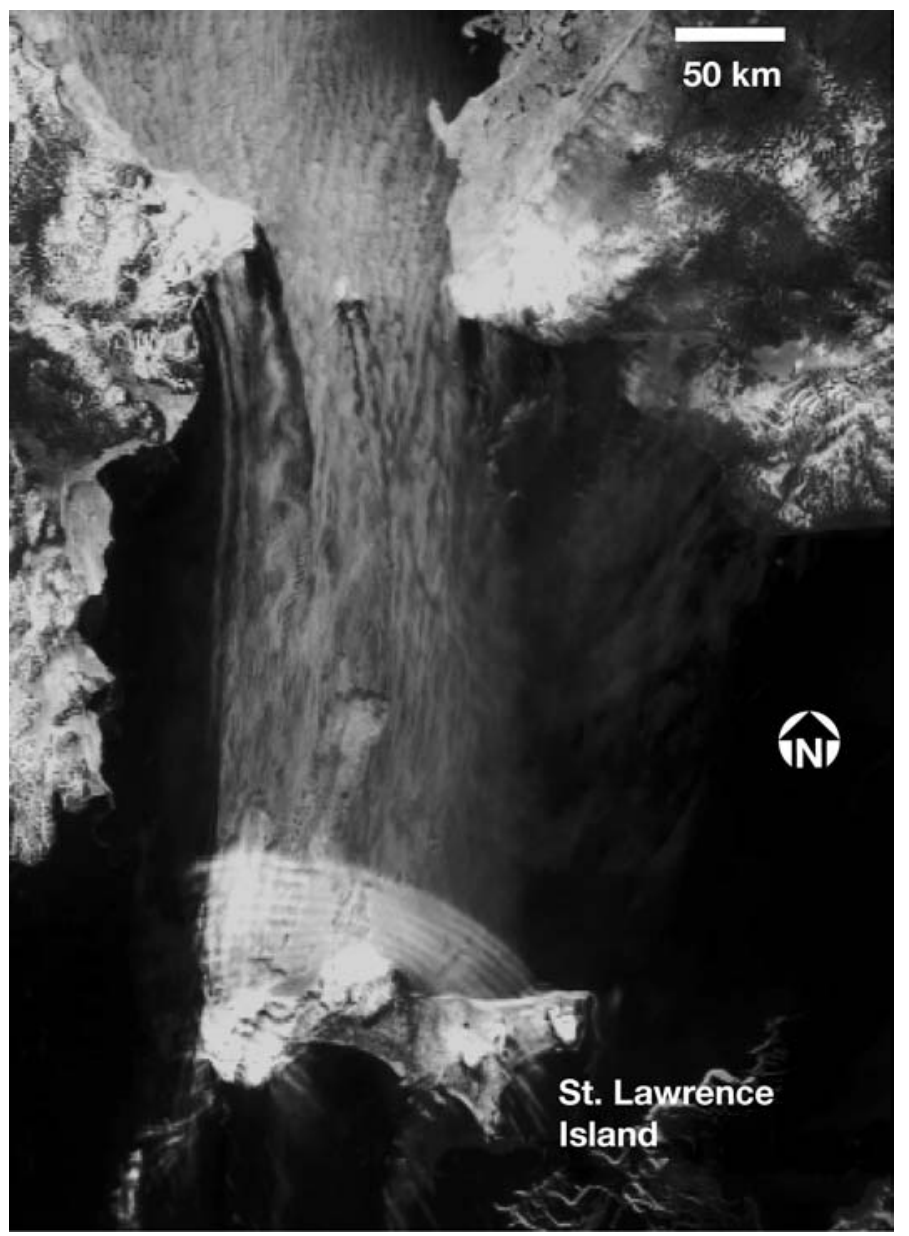

\subsection{Weakly Nonlinear Models}

The $\mathrm{KdV}$ equation arises from an assumption that nonlinearity, scaled by $\alpha=a / H$, and nonhydrostatic dispersion, $\beta=(H / l)^{2}$, are comparable and small: $\beta=O(\alpha) \ll 1$. Here $a$ is a measure of the wave amplitude, $H$ is an intrinsic vertical scale, and $l$ is a measure of the wavelength. It may be that the waves are long with respect to $H$, say the depth of one layer, but not to the total depth. In that case, weakly nonlinear theories for infinitely deep fluids (Benjamin 1967, Ono 1975) or intermediate depth (Joseph 1977, Kubota et al. 1978) are available. However, we focus on the $\mathrm{KdV}$ model as it has been used widely and is appropriate in many situations of interest.

A useful variant of the $\mathrm{KdV}$ equation is the extended $\mathrm{KdV}(\mathrm{eKdV})$ equation, which includes cubic nonlinearity (Djordjevic \& Redekopp 1978, Kakutani \& Yamasaki 
1978, Lee \& Beardsley 1974):

$$
\eta_{t}+\left(c_{0}+\alpha_{1} \eta+\alpha_{2} \eta^{2}\right) \eta_{x}+\beta_{1} \eta_{x x x}=0 .
$$

Here the wave amplitude $\eta(x, t)$ is related to the isopycnal vertical displacement, $t$ is time, and $x$ is the spatial variable in the direction of wave propagation. The coefficients $\alpha_{1}, \alpha_{2}$, and $\beta_{1}$ are functions of the steady background stratification and shear through the linear eigenmode (vertical structure function) of interest. The linear phase speed $c_{0}$ is the eigenvalue of the Sturm-Louiville problem for the eigenmode.

For a two-layer system with a rigid lid and no mean flow, in the Boussinesq approximation,

$$
\begin{aligned}
c_{0} & =\left(\frac{g \sigma b_{1} b_{2}}{b_{1}+b_{2}}\right)^{1 / 2}, \\
\alpha_{1} & =\frac{3}{2} c_{0} \frac{b_{1}-b_{2}}{b_{1} b_{2}}, \\
\alpha_{2} & =\frac{3 c_{0}}{\left(b_{1} b_{2}\right)^{2}}\left[\frac{7}{8}\left(b_{1}-b_{2}\right)^{2}-\left(\frac{b_{2}^{3}+b_{1}^{3}}{b_{1}+b_{2}}\right)\right], \\
\beta_{1} & =\frac{c_{0}}{6} b_{1} b_{2} .
\end{aligned}
$$

Here $g$ is the gravitational acceleration, $\sigma=2\left(\rho_{2}-\rho_{1}\right) /\left(\rho_{1}+\rho_{2}\right) \ll 1$ is the relative layer density difference, $\rho_{1}\left(\rho_{2}\right)$ is the density of the upper (lower) layer, and $b_{1}$ and $h_{2}$ are the mean upper and lower layer depths, respectively. In this case, $\eta(x, t)$ is the departure of the interface from the mean position. General relations for the coefficients with continuous stratification and shear can be found in, for example, Lamb \& Yan (1996) or Grimshaw et al. (2002).

The cubic term in Equation 1 is $O\left(\alpha^{2}\right)$, and thus Equation 1 should formally include additional $O\left(\beta^{2}\right)$ and $O(\alpha \beta)$ terms to give a higher-order $\mathrm{KdV}$ equation valid to $O\left(\alpha^{2}\right)$ (Grimshaw et al. 2002, Koop \& Butler 1981, Lamb \& Yan 1996). However, if the background stratification gives $\alpha_{1}=O(\alpha)$, as occurs in the two-layer case when $\left|h_{1}-b_{2}\right| /\left(b_{1}+b_{2}\right) \ll 1$, then the $\mathrm{eKdV}$ equation is asymptotically consistent, but requires the balance $\beta=O\left(\alpha^{2}\right)$.

Solitary wave solutions to the $\mathrm{eKdV}$ equation (Equation 1) can be written in a number of forms (Kakutani \& Yamasaki 1978, Miles 1979, Ostrovsky \& Stepanyants 1989), one of which is

$$
\eta=\frac{\eta_{0}}{b+(1-b) \cosh ^{2} \gamma(x-c t)}
$$

where

$$
c=c_{0}+\frac{\eta_{0}}{3}\left(\alpha_{1}+\frac{1}{2} \alpha_{2} \eta_{0}\right), \quad \gamma^{2}=\frac{\eta_{0}\left(\alpha_{1}+\frac{1}{2} \alpha_{2} \eta_{0}\right)}{12 \beta}, \quad b=\frac{-\eta_{0} \alpha_{2}}{2 \alpha_{1}+\alpha_{2} \eta_{0}} .
$$

Here $\eta_{0}$ is the wave amplitude and $b$ is a parameter.

When $\alpha_{2}$ is set to zero Equation 1 reduces to the $\mathrm{KdV}$ equation and the classical sech $^{2}$ solution is recovered from Equations 6 and 7. The solitary wave solutions require $\eta_{0} \alpha_{1}>0$. Thus, in the two-layer Boussinesq system, the solitary waves are 
Figure 4

Examples of solitary wave solutions of the eKdV equation (Equation 6) for the arbitrary choice of the parameters

$\beta=\alpha_{1}=-\alpha_{2}=1$. As the maximum wave amplitude increases, the waves eventually broaden and develop a flat crest at the maximum amplitude $\eta_{0 \max }=1$.

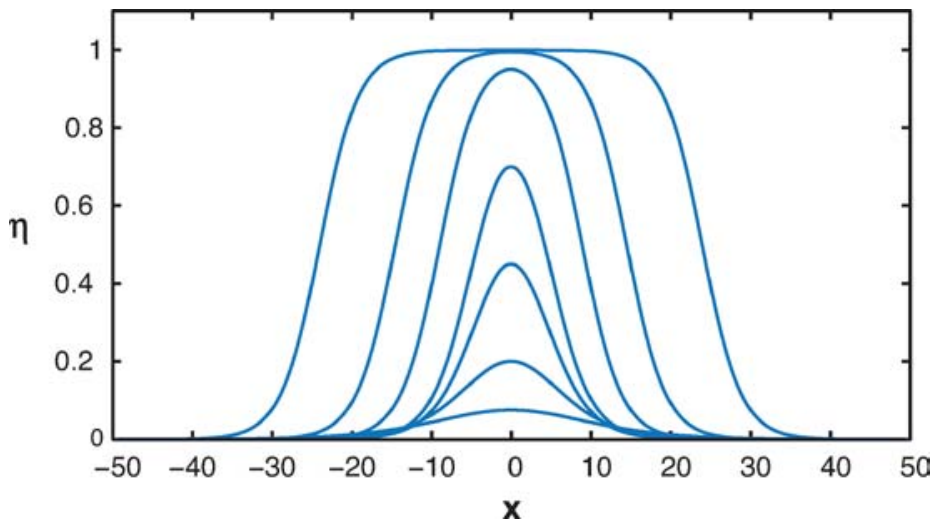

waves of elevation (depression) for $h_{1} / h_{2}>1(<1)$. The wavelength, $\lambda \sim\left|\eta_{0}\right|^{-1 / 2}$, decreases with increasing amplitude and does not capture the broadening of waves that is often observed (see Figure $2 \boldsymbol{b}$ ).

In contrast, eKdV solitary waves capture this shape when $\alpha_{2}<0(0<b<1)$. Small-amplitude waves become narrower with increasing $\left|\eta_{0}\right|$, as do KdV solutions. However, as the upper limit $(b \rightarrow 1)$ is approached the waves begin to broaden until a wave of maximum amplitude $\eta_{0 \max }=-\alpha_{1} / \alpha_{2}$ is reached. Figure 4 shows a plot of eKdV solitary waves with a range of amplitudes for $\beta=\alpha_{1}=-\alpha_{2}=1$ and $\eta_{0 \max }=1$. The maximum wave becomes infinitely long and consists of a broad plateau terminated at each end by dissipationless bores, $\eta=\frac{1}{2} \eta_{0 \max }\left(1 \pm \tanh \gamma_{\max }\left(x-c_{\max } t\right)\right)$. The bores travel at the maximum speed $c_{\max }=c_{0}-\alpha_{1}^{2} /\left(6 \alpha_{2}\right)$. The mass, momentum, and energy in the steady frame moving at $c_{\max }$ are conserved (at the order of Equation 1) across a bore. Thus, the flows on either side are "conjugate states" (Benjamin 1966).

An illustration of the departure of the $\mathrm{eKdV}$ model from the $\mathrm{KdV}$ theory is given in Figure 5. The amplitude dependence of the wave speed $c$ and wavelength, $L_{w}=\eta_{0}^{-1} \int_{-\infty}^{\infty} \eta d x^{1}$, from the $\mathrm{KdV}$ and $\mathrm{eKdV}$ models are plotted for the two-layer stratification with $b_{1} / h_{2}=1 / 4$ and $h_{1} / h_{2}=2 / 3$. Significant differences between the $\mathrm{eKdV}$ and $\mathrm{KdV}$ solutions emerge for relatively small amplitudes.

For more general stratifications and background shear flows the coefficient of the cubic term $\alpha_{2}$ can have either sign. When it is positive, solitary wave solutions still exist, but now the waves may have either polarity regardless of the sign of $\alpha_{1}$ (Grimshaw et al. 1997b, 2004). However, the broadening character and upper bound on wave amplitude are lost and replaced by a minimum wave amplitude $\eta_{0}=-2 \alpha_{1} / \alpha_{2}$ for $\alpha_{1} \eta_{0}<0$. Considering the evolution of long sine waves in a three-layer stratification with $\alpha_{2}>0$, Grimshaw et al. (1997b) find that solitary waves of both polarities may evolve simultaneously for either sign of $\alpha_{1}$.

\footnotetext{
${ }^{1}$ Here $L_{w}=2 \gamma^{-1}$ for $\mathrm{KdV}$ solitary waves.
} 


$$
h_{1} / h_{2}=0.25
$$
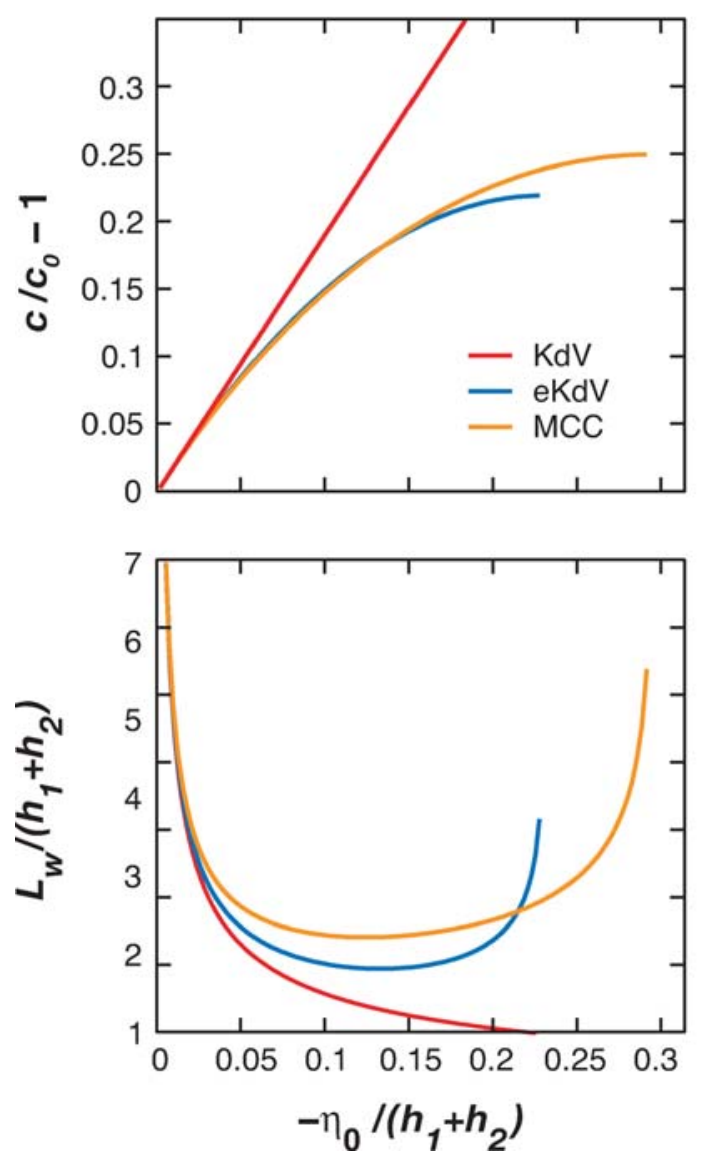

$h_{1} / h_{2}=2 / 3$
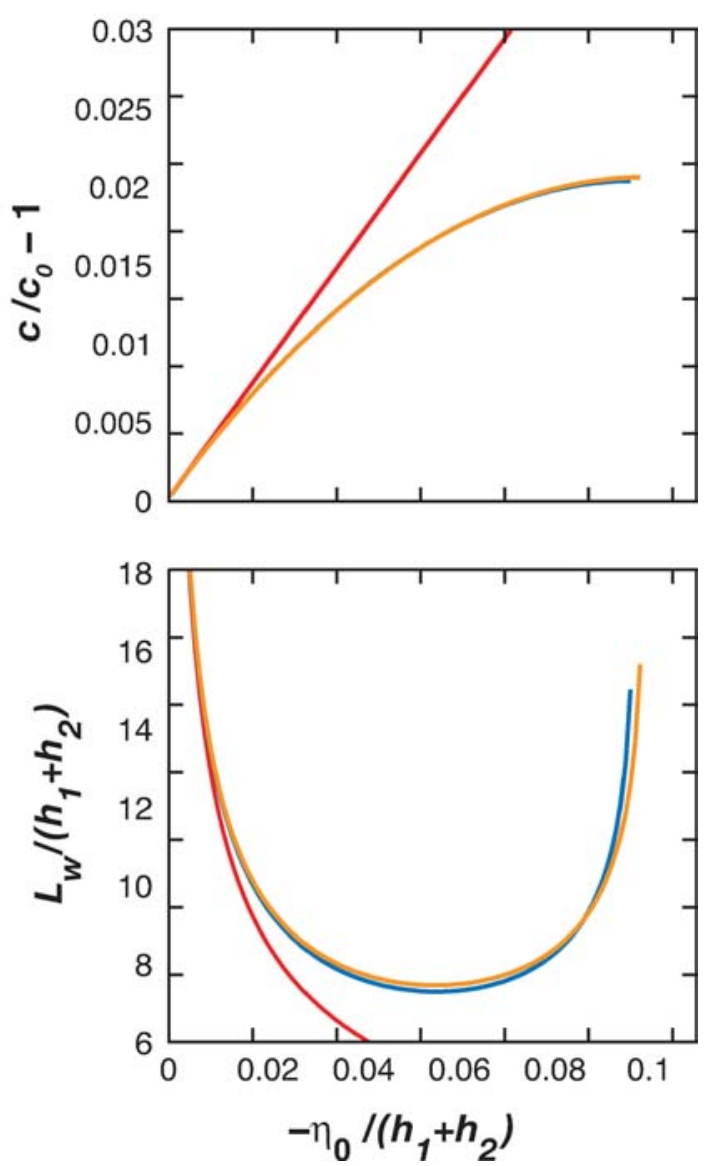

Figure 5

Comparison of solitary wave properties from the two-layer KdV (red), eKdV (blue), and MCC (orange) theories. The top row shows the wave speed $c$ vs. amplitude $\eta_{0}$ and the bottom row shows the wavelength $L_{w}$ vs. $\eta_{0}$. The comparison is done for the two stratifications $h_{1} / h_{2}=1 / 4$ (left column) and $h_{1} / h_{2}=2 / 3$ (right column). For both the eKdV and MCC waves the maximum wave amplitude corresponds to the end of the speed curves.

\subsection{Large-Amplitude Models}

The $\mathrm{KdV}$-type theories have been used with some success to model wave evolution outside their formal range of validity (Holloway et al. 1997, 1999). Surprisingly, Stanton \& Ostrovsky (1998) found that the eKdV theory did a good job of capturing the characteristics of the highly nonlinear waves they observed (c.f., Figure $2 \boldsymbol{b}$ ). Thus, it has been adopted as the phenomenological model of choice. However, finiteamplitude theories are ultimately required to accurately describe properties of oceanic observations of waves with $\alpha=O(1)$. 
2.2.1. Long wave theories. A useful extension of the weakly nonlinear two-layer eKdV model was proposed by Miyath $(1985,1988)$ and Choi \& Camassa (1999). They each derived equivalent two-layer models with full nonlinearity, $\alpha=O(1)$, while retaining only the first-order weakly dispersive effects, $\beta \ll 1$. The result is a coupled set of bi-directional wave equations that in the limit $\beta \rightarrow 0$ reduce to the two-layer shallow-water equations. The solitary wave solutions of the MiyataChoi-Camassa (MCC) equations broaden and slow (relative to $\mathrm{KdV}$ ) with increasing amplitude. The theory produces a maximum wave with amplitude $\eta_{0 \max }=\left(b_{1}-b_{2}\right) / 2$ (in the rigid-lid Boussinesq limit, $\sigma \ll 1$ ) that reaches mid-depth and also has infinite wavelength (conjugate states). Comparison of the wave shapes and properties shows that the $\mathrm{eKdV}$ and MCC theories agree quite well for $0.4<b_{1} /\left(b_{1}+b_{2}\right)<0.6$, where the scaling requirements of $\mathrm{eKdV}$ are reasonably met. However, differences grow rapidly outside this range, as illustrated in Figure 5. MCC solitary wave solutions are in good agreement with the laboratory experiments (Choi \& Camassa 1999, Michallet \& Barthelemy 1998), the full numerical solutions to the Euler equations, and observations (Ostrovsky \& Grue 2003) over a wide range of relative layer depths. However, the MCC equations have a potential limitation as a modeling tool. Jo \& Choi (2002) found that solitary waves of sufficient amplitude could be unstable at high wave numbers to Kelvin-Helmholtz instability. If the grid resolution was too fine, unstable short waves first emerged near the wave crest and ultimately overwhelmed the calculations.

Ostrovsky \& Grue (2003) derived equations equivalent to the MCC equations for strongly nonlinear dispersive waves. Using Riemann invariants, they added nonlinear dispersive effects from both the MCC formulation and phenomenologically to arrive at unidirectional evolution equations for strongly nonlinear dispersive waves that are generalizations of the $\mathrm{KdV}$ and Benjamin-Ono equations. For example, the phenomenological derivation of the $\mathrm{KdV}$ equation gives

$$
\eta_{t}+c(\eta) \eta_{x}+\left(\beta(\eta) \eta_{x x}\right)_{x}=0
$$

where $c(\eta)$ is the exact nonlinear speed from the nondispersive theory and

$$
\beta(\eta)=\frac{1}{6} c(\eta)\left(b_{1}+\eta\right)\left(b_{2}-\eta\right)
$$

is the generalization of the coefficient of $\mathrm{KdV}$ dispersion term (Equation 5).

These equations retain solitary wave solutions with properties that agree reasonably well with the MCC equations, with full numerical solutions, and with the observations of Stanton \& Ostrovsky (1998). These equations avoid the instability found for the MCC equations. However, none of these models have been studied much beyond the properties of the solitary wave solutions. The exception is Jo \& Choi (2002), who considered collisions between solitary waves and several examples of large-amplitude solitary wave evolution over slowly varying topography with the MCC theory.

2.2.2. Fully nonlinear waves. The restriction to weakly nonlinear and/or weakly nonhydrostatic conditions can be avoided through solutions of the Euler equations 
for steady solitary waves. Two-layered systems have been considered in detail in numerous studies (e.g., Evans \& Ford 1996, Funakoshi \& Oikawa 1986, Grue et al. 1999, Pullin \& Grimshaw 1988, Turner \& Vanden-Broeck 1988). Potential flow within each layer allows the full formulation to be reduced to boundary integral equations. The solitary wave solutions both broaden with amplitude and reach a limiting height. In a Boussinesq fluid, the limiting flat-crested wave has the same amplitude $\eta_{0 \max }=\left(b_{1}-b_{2}\right) / 2$ found in the MCC theory. MCC solitary waves are nearly indistinguishable from the fully nonlinear theory over a wide range of relative layer depths (Michallet \& Barthelemy 1998). Thus, the long wave limitation of the MCC theory is not overly restrictive in predicting the properties of individual waves.

Solitary waves in continuously stratified flows have been explored with numerical solutions of the Dubriel-Jacotin-Long (DJL) equation (Long 1953). This approach originated with Benjamin (1966), Davis \& Acrivos (1967), and Tung et al. (1982), who discussed properties of mode-two solitary waves in deep fluids. More recent examples are the studies of Turkington et al. (1991) and Brown \& Christie (1998), who sought mode-one solitary wave solutions. Depending on the backgound density field, expressed as $\rho=\rho(\psi)$, where $\psi$ is the streamfunction, the solitary wave amplitude is limited in one of three ways (Lamb 2002). If the density gradient is zero at the boundary of the shallow layer, and the pycnocline is relatively broad and not too close to a boundary, the wave amplitude is limited by a conjugate flow. If the pycnocline is sharper, or close to a boundary, the solutions can be found for amplitudes increasing until the Richardson number drops to 0.25 at some location within the flow. Lamb (2002) termed this a stability limit, although he points out that this does not necessarily imply that the waves are unstable, or that stable waves with lower Richardson numbers do not exist. In any case, the issue of wave stability cannot be addressed with a steady model. The third limiting situation occurs when the density gradient is nonzero at the boundary under the crest (the top surface for a wave of depression); the wave may develop vertical streamlines and particle velocities (at the surface) equal to the wave phase speed, indicating incipient overturning and breaking (Fructus \& Grue 2004, Grue et al. 2000, Lamb 2002).

The solutions can be continued beyond this point if $\rho(\psi)$ is specified a priori. Typically, the background relation is used, giving solutions with closed streamlines (in a frame moving with the wave) and a recirculating vortex core. Thus, these waves transport an isolated volume of fluid. However, this choice for $\rho(\psi)$ leads to density inversions, and, most likely, static instability. Additionally, the densities within the core are outside the range of the background state. Just how to specify $\rho(\psi)$ beyond the overturning limit is unclear. It should depend on the unsteady processes that lead to wave formation, gravitational instability within the core, and diffusive processes that ultimately homogenize the density and couple the core circulation to the exterior flow. All are beyond the scope of a steady inviscid theory. Lamb (2002) used timedependent numerical solutions to show that topographically induced breaking of a shoaling solitary wave with open streamlines could result in the formation of a modeone wave with a recirculating trapped core. The limiting wave crests were nearly flat and reminiscent of the conjugate states. This led Lamb \& Wilkie (2004) to develop 
a theory for conjugate flows with trapped cores with uniform density, and specified core vorticity, that agreed quite well with the transient calculations.

Extensions of DJL solutions to accommodate a mean background shear showed that wave amplitudes are limited by the same three situations just described (Lamb 2003, Stastna \& Lamb 2002). However, the wave properties were sensitive to the background vorticity. For example, background vorticity near the surface, of the same sign as the wave-induced vorticity, could induce wave breaking in stratifications that without shear would give a conjugate state.

When the stratification is uniform and Boussinesq, the nonlinear terms in the DJL equation are identically zero. This corresponds to $\alpha_{1}=0$, for which $\mathrm{KdV}$ solitary waves do not exist. If the stratification is allowed to be slightly nonuniform, or non-Boussinesq, then steady solitary waves can again be found by balancing weak nonlinearity against weak dispersion (Benney \& Ko 1978, Grimshaw \& Yi 1991), with the necessary balance achieved by finite-amplitude waves. Solitary waves exist up to an amplitude limited by incipient breaking. Derzho \& Grimshaw (1997) determined properties of steady solitary waves beyond this point by assuming the presence of a constant density and, at lowest order, an irrotational core. The presence of the core causes the waves to broaden and slow compared to the waves below the threshold.

\subsection{Laboratory Experiments}

Weakly nonlinear $\mathrm{KdV}$ theories have been shown in a number of studies to have a robust range of validity when compared to laboratory experiments for individual solitary wave properties (Grue et al. 1999, Koop \& Butler 1981, Michallet \& Barthelemy 1998, Segur \& Hammack 1982). Koop \& Butler (1981) and Segur \& Hammack (1982) showed that, for weakly nonlinear waves in deep water, the KdV model is quantitatively better than the Benjamin-Ono theory. In a series of experiments in a two-layer fluid with $b_{1} / b_{2}=0.24$, Grue et al. (1999) found that KdV theory was good for wave amplitudes as large as $\eta_{0} / h_{1} \approx 0.4$. However, the KdV model does not capture the eventual broadening and slowing of solitary waves with increased amplitude. Higher-order $\mathrm{KdV}$ theories improve the situation somewhat for $b_{1} / b_{2} \ll 1$ (Koop \& Butler 1981), but ultimately also fail for the same reasons. The eKdV theory offers some improvement, especially for $\alpha_{1} \ll 1$. Michallet $\&$ Barthelemy (1998) found excellent agreement with two-layer eKdV theory and experiments for $0.4<b_{1} /\left(b_{1}+b_{2}\right)<0.6$.

The experiments of Michallet \& Barthelemy (1998) and Grue et al. (1999) also demonstrated that fully nonlinear two-layer theories are in excellent agreement with laboratory measurements of solitary wave properties over a wide range of relative layer depths. Grue et al. (1999) observed that waves approaching the limiting amplitude exhibited an instability on the rear face of the wave. The shape of the leading face agreed very well with the theory, whereas the instability led to mixing and an asymmetric broadening of the wave. Estimated interfacial Richardson numbers near the wave crest were less than 0.25 for one unstable case and greater than this for three stable cases. 
Davis \& Acrivos (1967), Maxworthy (1980), and Stamp \& Jacka (1996) considered mode-two waves produced by the gravitational collapse of mixed fluid into a stratified layer bounded by deep homogeneous layers. Small-amplitude wave properties agreed with the Benjamin-Ono weakly nonlinear theory, whereas large-amplitude waves developed trapped cores that slowly leaked mass out behind the wave. Grue et al. (2000) considered first-mode waves in an extensive set of experiments in twolayer flows with a uniformly stratified upper. For small and moderate wave amplitudes $a / b_{1}<0.5\left(b_{1} / b_{2}=4.13\right)$, the wave shapes, speeds, and velocity profiles under the wave crests were in good agreement with the models. However, wave breaking in the form of small vortices was observed near the free surface (for waves of depression) in the leading part of the wave at $a / b_{1} \approx 0.6$. Large amplitudes produced more vigorous breaking and broadening of the wave beyond the theoretical predictions. Fluid velocities in a region above the crest were equal to the phase speed, possibly indicating a trapped core. Thus, Grue et al. (2000) attributed the instability to incipient overturning. However, the quoted errors in the velocity measurements and lack of imagery in both sets of experiments leave the origin of the observed instabilities unclear.

\section{WAVE EVOLUTION}

While waves of permanent form, including solitary waves, are useful for the approximate interpretation of observations of nonlinear internal waves, the neglect of time dependence necessarily excludes the processes of wave generation, evolution, and, finally, dissipation.

\subsection{Generation}

With the early evidence of the tidal forcing of packets of internal waves and the competing effects of nonlinearity and dispersion, Lee \& Beardsley (1974) recognized that an inhomogeneous eKdV-type model (based on the earlier formulation of Benney 1966), including forcing due to the barotropic tidal flow over topography, should capture the essential elements of wave generation. Their numerical and laboratory results, and the field observations of Halpern (1971), were interpreted in the context of three components: initial generation of a front upstream due to "blocking" by topography, nonlinear steepening of the front, and finally, the generation of a wave packet by the combined effects of nonlinearity and dispersion.

Maxworthy (1979) questioned this model with regard to the phase of the response to the tidal forcing. On the basis of laboratory experiments, he suggested that the observed shoreward-propagating waves were generated when the tide changed from ebb (offshore) to flood (onshore), releasing the lee wave formed on the ebb tide to propagate back over the topography and evolve through $\mathrm{KdV}$ dynamics into a rank-ordered packet of waves. In the case where the ebb tide was strong enough to lead to significant mixing, he suggested that the subsequent relaxation of the mixed region when the tide turned could force first-mode internal waves on both sides of 
the topography. In fact, depending on conditions, both Lee \& Beardsley's (1974) and Maxworthy's (1979) mechanisms may apply (also see Matsuura \& Hibiya 1990).

Some of the elements of tidal generation are contained in the simpler problem of transcritical stratified flow over topography, which replaces the periodic tidal flow with steady flow far upstream (see Figure 3). For weak nonlinearity, dispersion, and forcing, the canonical equation is the forced $\mathrm{KdV}(\mathrm{fKdV})$ equation, which was derived by Grimshaw \& Smyth (1986) for continuous stratification. Melville \& Helfrich (1987) derived the forced $\mathrm{eKdV}$ equation and compared solutions with laboratory experiments in a two-layer flow of immiscible fluids over topography. For the $\mathrm{fKdV}$ equation, their solutions showed the previously seen (Grimshaw \& Smyth 1986) progression of solutions from undular bores, to sequences of solitary waves in a transcritical regime, to locally steady flow over the topography at supercritical Froude numbers. Due in part to the relatively strong forcing, Melville \& Helfrich (1987) found poor agreement between the numerical solutions and experiments. In contrast, for the cases in which the cubic nonlinearity became important, the agreement between measurements and the numerical solutions was improved, showing a progression from undular bores upstream, to monotonic bores in the transcritical regime, to steady flow over the topography for supercritical flows (see Figure 6).

Grue et al. (1997) developed a numerical scheme for fully nonlinear two-layer systems and found very good agreement with the measurements of Melville \& Helfrich (1987). They concluded that weakly nonlinear theories may have quite limited application in modeling unsteady transcritical two-layer flows, and that fully nonlinear methods are generally required.

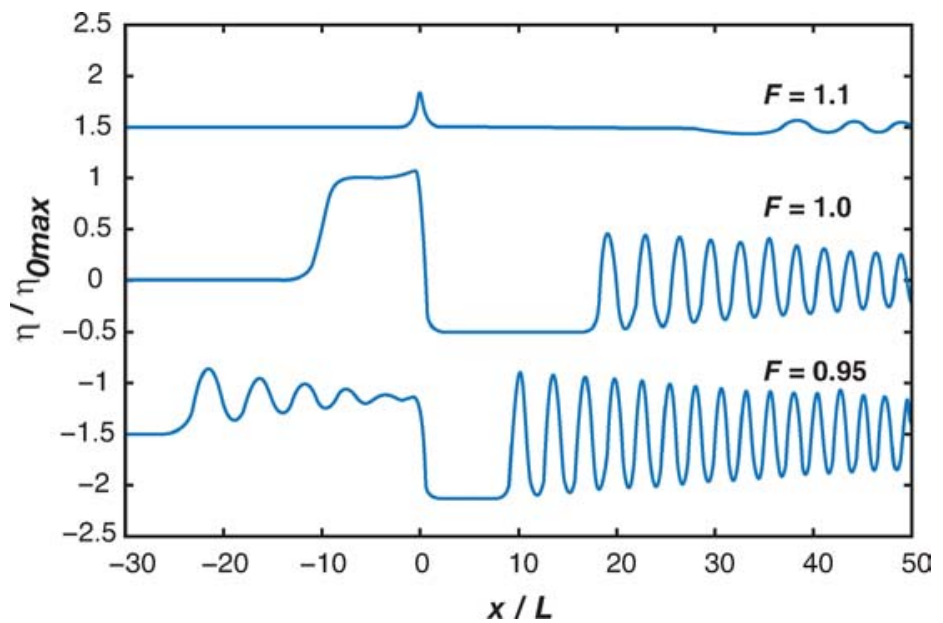

Figure 6

Three solutions of the forced eKdV equation in the transcritical regime showing the transition from upstream propagating undular bore $(F=0.95)$, to upstream monotonic bore $(F=1)$, to stationary supercritical flow $(F=1.1)$. The topography is a Gaussian bump located at $x=0$ with length scale $L$. The solutions are shown at the same time after initiation of the forcing and for the same stratification and other parameters as in figure 3 of Melville \& Helfrich (1987). 
Grimshaw \& Yi (1991) addressed the case of a uniform weakly stratified Boussinesq flow over topography, in which case the quadratic nonlinearity goes to zero. The resulting solutions have some similarity to those of the $\mathrm{fKdV}$ equation but now weak forcing produces an $\mathrm{O}(1)$ response and the wave amplitudes are limited by breaking, defined as an incipient flow reversal. Their evolution equation reduces to the $\mathrm{fKdV}$ equation for small wave amplitudes.

In a field study of the generation of internal waves upstream of a sill in Knight Inlet, Farmer \& Armi (1999) showed acoustic images of the evolution of the flow that suggest that the deepening thermocline that plunges and accelerates the flow in the lower layer over the sill can become unstable to shear flow instabilities that may propagate upstream and evolve into (or generate) a train of solitary internal waves. The extensive Knight Inlet data set has aroused the interest of numerical modelers (see Lamb 2004, Stastna \& Peltier 2004), and has led to some controversy regarding the relative merits of simple quasi-steady hydraulic models and the predictions and interpretations of time-dependent numerical models. What does not appear to be controversial is that strongly nonlinear solitary-like waves are generated upstream, even in flows that contain significant turbulence and mixing in the neighborhood of the topography. Furthermore, $\mathrm{KdV}$ dynamics leads to the existence of an intrinsically unsteady transcritical regime associated with upstream wave generation and propagation (Grimshaw \& Smyth 1986, Melville \& Helfrich 1987). Stastna \& Peltier (2004) suggest that the resonant generation of upstream waves in the simpler models carries through to flows over more complex topography.

\subsection{Evolution}

Much of the observational literature on long nonlinear internal waves has been dominated by the fact that asymptotic solutions to the conservative evolution equations show the emergence of solitary waves after a sufficiently long time. The question is: how long a time? Furthermore, in nature, a number of complicating factors may conspire to influence the evolution of the waves from the generation site. These include nonconservative effects associated with dissipation in boundary layers, radiation damping through coupling with other wave modes, scattering at the boundaries, and modulations due to inhomogeneities in the stratification and large-scale currents. A complete review of all of these effects is beyond the space available here; however, we touch on some of them.

Hammack \& Segur (1978) considered the different temporal regimes for solutions to the $\mathrm{KdV}$ equation for surface waves, but their results are easily extended to internal waves (Helfrich 1984). The results for a two-layer system are based on the two parameters

$$
V_{0}=\frac{3}{2} \frac{\left(b_{1}-h_{2}\right)}{\left(h_{1}+h_{2}\right)^{3 / 2}} L_{0} a_{0}, \quad U_{0}=\frac{L_{0}}{\left(b_{1} h_{2}\right)^{1 / 2}}\left|V_{0}\right|,
$$

where $a_{0}, L_{0}$ are the amplitude and length scales, respectively, of the initial disturbance. $V_{0}$ is the volume scale and $U_{0}$ is the Ursell number for the initial disturbance. Consider the case in which $b_{1}<b_{2}$ with $a_{0}<0$, then $V_{0}>0$, and if $U_{0} \gg 1, \mathrm{KdV}$ 
dynamics applies immediately and the leading solitary wave emerges after a time

$$
t_{s} \approx 6 \frac{U_{0}^{2}}{V_{0}^{3}} T, \quad T=\left(\frac{b_{1}+b_{2}}{g \sigma}\right)^{1 / 2} .
$$

Applying these criteria to the observations of Halpern (1971) in Massachusetts Bay, the sorting time for solitary waves is approximately $5 \times 10^{4} \mathrm{~s}$ (14 hours) over a propagation distance of approximately $30 \mathrm{~km}$, comparable to the width of Massachusetts Bay. For the waves observed in the Andaman Sea by Osborne \& Burch (1980), the sorting time and distance would be $5 \times 10^{5} \mathrm{~s}$ (140 hours) and $1200 \mathrm{~km}$, respectively (Helfrich 1984). In the latter case, the sorting distance is comparable to the largest observed propagation distance of $900 \mathrm{~km}$. However, for the shortest possible distance of $300 \mathrm{~km}$, the waves are not likely fully sorted.

3.2.1. Variable topography or stratification. For KdV-like models of slowly varying waveguides, much of the literature for long surface-wave evolution carries over and is not reviewed here (see Miles 1980). However, there is an important difference for internal waves, which is associated with the fact that the coefficient of the quadratic term may change sign at a "turning point," which in the two-layer case with a small density difference between the layers corresponds to the point where $b_{1}=b_{2}$.

Knickerbocker \& Newell (1980) considered the problem using a KdV equation with slowly varying coefficients and argued that as the solitary wave propagates up the slope it would develop a lengthening trailing shelf of opposite sign. On approaching the turning point, the solitary wave deformed and lengthened, then, on passing through the turning point, waves of elevation evolved from the trailing shelf.

For slowly varying topography the waves may be in the neighborhood of the turning point for some considerable time and the effects of cubic nonlinearity may dominate quadratic nonlinearity, limiting the amplitude of solitary waves (Long 1956). Helfrich et al. (1984) formulated the eKdV equation for slowly varying topography ( $l / L=O(\alpha)$, where $L$ is the horizontal length scale of the topography) and computed numerical solutions for solitary waves of depression propagating over slope-shelf topography, finding qualitative agreement with the essential features of the numerical solution of Knickerbocker \& Newell (1980). They also used the numerical solutions at the top of the shelf, along with the Miura transformation and inverse scattering theory (Ablowitz \& Segur 1981), to determine the asymptotic solution on the shelf, especially the number and amplitudes of the solitary waves.

However, in attempting to confirm the solutions of the inviscid eKdV model with laboratory experiments, Helfrich \& Melville(1986) found it necessary to include the effects of viscous boundary layers (Grimshaw 1981, Kakutani \& Matsuuchi 1975, Miles 1976), which were as significant as the nonlinear and dispersive effects in their $\mathrm{eKdV}$ model. Numerical solutions showed fair to good agreement with the evolution of the leading waves over slope-shelf topography in the laboratory, but in no case were waves of elevation observed on the shelf, a fact attributed to the strong damping and relatively fast slopes of the laboratory experiments. However, the experiments also demonstrated that shear instabilities at the interface, and kinematic breaking, could occur in the vicinity of the shelf break, with the latter leading to regions of mixed fluid 
on the shelf. In some cases the mixed fluid evolved into, or forced, what appeared to be second-mode solitary waves (c.f., Davis \& Acrivos 1967, Maxworthy 1980, Vlasenko \& Hutter 2001). Grimshaw et al. (2004) also explored the turning point problem in waveguides where the coefficient of the cubic nonlinearity $\alpha_{2}$ changes sign.

Observations by Klymak \& Moum (2003) show evidence of waves of elevation in a bottom stratified layer off the coast of Oregon. Liu et al. (1998) report that phase shifts seen in the SAR images of internal waves in the South China Sea are evidence of a change of polarity. Perhaps the best direct evidence so far also comes from the South China Sea. Orr \& Mignerey (2003) show acoustic images of internal waves with significant instabilities associated with the passage of waves of depression across the shelf break, but also evidence of waves of elevation emerging on the shoreward side. Figure 7 from Lynch et al. (2004) (see also Ramp et al. 2004) shows evidence of the evolution of a packet of waves of depression in deeper water evolving into waves of elevation in shallower water.

3.2.2. Effects of rotation. Rotational effects may be comparable to weak nonlinear and dispersive effects if the Rossby number, $R_{o}=c_{0} / f l \gg 1$, where $f$ is the Coriolis parameter $\approx \pm 10^{-4} \mathrm{rad} / \mathrm{s}$ at mid latitudes. Ostrovsky (1978) extended the unidirectional $\mathrm{KdV}$ equation to include the effects of weak rotation on nonlinear dispersive internal waves. Odolu (1978) (also see Grimshaw 1985) extended the formulation to include weak transverse effects to obtain a generalization of the Kadomtsev \& Petviashvili (1970) equation, the rotation-modified KP equation, which in scaled form becomes

$$
\left(\eta_{t}+\frac{3}{2} \eta \eta_{x}+\frac{1}{6} \eta_{x x x}\right)_{x}+\frac{1}{2}\left(\eta_{y y}-\eta\right)=0,
$$

where

$$
\eta_{y}+\eta=0 \quad y=0, W
$$

corresponds to the no-flux condition through the sidewalls in a bounded domain.

When transverse variations are ignored $\left(\eta_{y y}=0\right)$ in Equation 8 , the equation is known as the rotating $\mathrm{KdV}$ equation and Leonov (1981) showed that it does not admit steady solitary waves. An initial KdV solitary wave will decay in finite time by radiation of Poincaré waves (Grimshaw et al. 1997a). Ostrovsky (1978) found that when the nonhydrostatic dispersion was also ignored $\left(\eta_{x x x}=0\right)$ in Equation 8, low-frequency rotational dispersion could balance nonlinearity and support periodic waves. However, the amplitude of these hydrostatic periodic waves has an upper bound beyond which rotational dispersion is too weak to balance nonlinearity.

In a study of internal tides, Gerkema \& Zimmerman (1995) and Gerkema (1996) found that the production of solitary-like waves could be inhibited by rotation. They formulated the two-layer weakly nonlinear Boussinesq equations with variable topography and considered forcing of internal tides for various regimes of nonlinearity, dispersion, and rotation. If the forcing was weak, or rotation strong, the radiated tide did not develop many high-frequency waves. In the opposite situation, the internal tide was eventually dominated by high-frequency solitary-like waves. The transition 


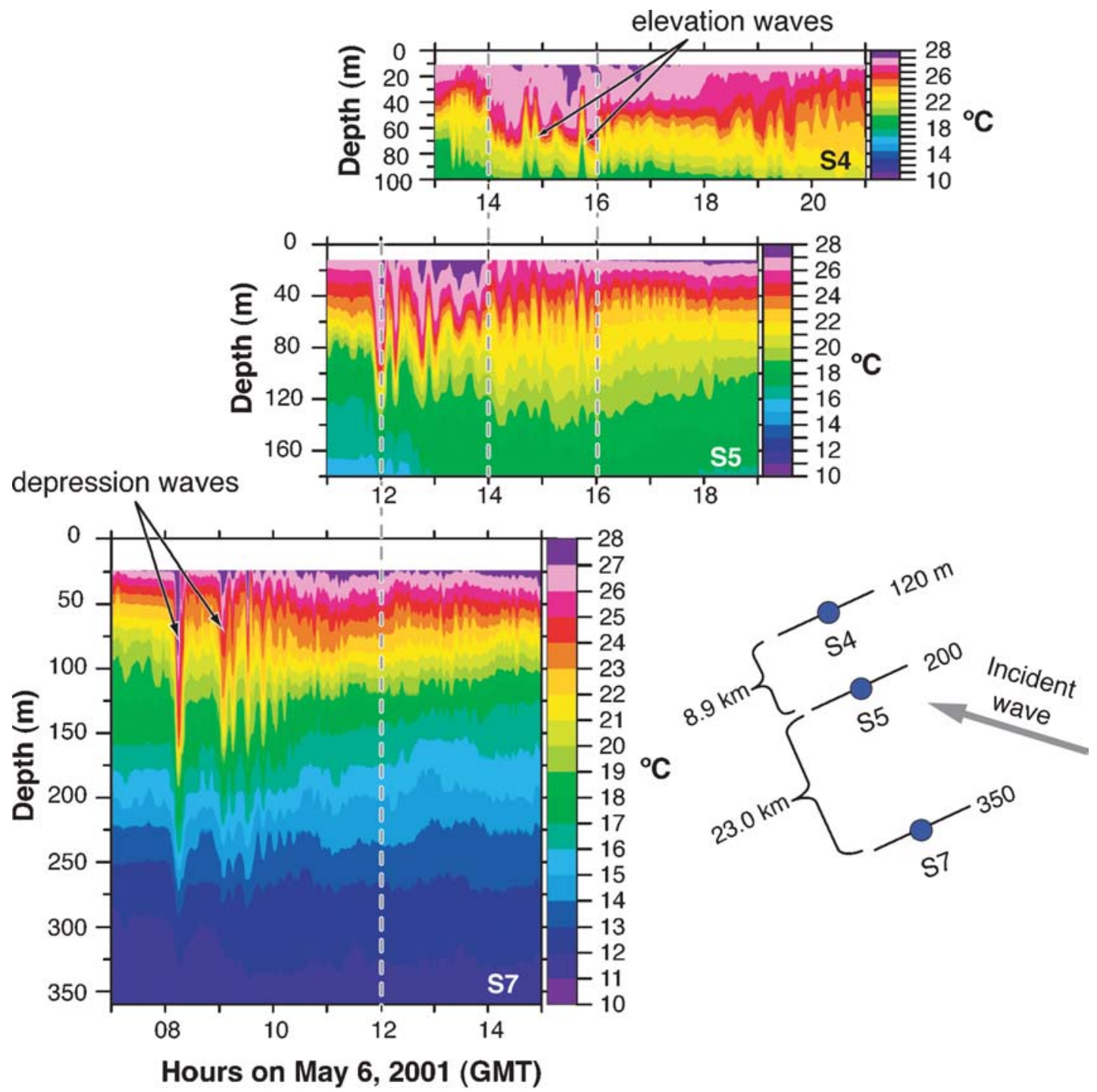

Figure 7

Temperature records from the South China Sea showing the transition from waves of depression to waves of elevation as the incident waves propagate into shallow water (from Lynch et al. 2004).

occurred for forcing nonlinearity (or internal tide amplitudes) comparable to the maximum allowable nonlinearity for the periodic hydrostatic waves.

The experiments of Maxworthy (1983) on nonlinear second-mode Kelvin waves in a channel drew attention to the fact that two hallmarks of linear Kelvin waves were modified by nonlinearity: The crests were curved (backward), and the transverse scale 
of the wave decay was not the Rossby radius of deformation $\left(c_{0} / f\right)$. Renouard et al. (1987) conducted larger-scale experiments on two-layer flows (first-mode waves) and confirmed Maxworthy's finding, but also found a train of small-amplitude waves in the wake of the leading Kelvin wave, which was slowly decaying along the channel. Katsis \& Akylas (1987) solved the rotation-modified KP equation for initial conditions corresponding to a straight-crested Kelvin wave normal to the rotating channel walls. Their solutions confirmed the curvature of the leading wave and the attenuation of the wave along the channel, which could not be due to viscous dissipation in this inviscid model.

Melville et al. (1989) formulated the equivalent single-layer problem in terms of a set of coupled evolution equations, which are asymptotically equivalent to a regularized version of the rotation-modified KP equation (Grimshaw \& Melville 1989). The scaled governing equation for the transverse ( $y$-component) velocity $v$, which is zero everywhere for linear Kelvin waves, becomes

$$
v_{t t}+v_{x t}-v_{x x t t}+\left(v-v_{y y}\right)=\left(1+\frac{\partial}{\partial y}\right)\left[u u_{x}\right],
$$

which is of the form $\mathcal{L}(v)=\mathcal{N}(u)$, where $\mathcal{L}$ is the linear operator for long linear dispersive waves in rotating systems, including linear Kelvin and Poincaré (inertiagravity) waves, and $u$ is the $x$-component of velocity. Equation 10 shows that weak transverse effects may be forced by the nonlinearities of the leading wave, especially in the neighborhood of resonances where the nonlinear speed of the leading wave matches the linear speed of the Poincaré waves. It is the superposition of the leading straight-crested Kelvin wave and the forced Poincare waves that can lead to the apparent curvature of the Kelvin waves. The radiation of the Poincaré waves also leads to the observed damping of the Kelvin wave (see also Akylas 1991, Grimshaw \& Melville 1989, Grimshaw \& Tang 1990).

The constraints that accompany the unidirectional approximation of the $\mathrm{KdV}$-type models are lost when bi-directional propagation is retained. Tomasson \& Melville (1992) used the rotation modified Boussinesq equations for the barotropic flow and showed that if the timescale for the effects of nonlinearity to be significant are shorter or comparable to the sorting time of the linear modes, then the free and forced Poincaré modes of the system may not be separated.

Other examples of nonlinear wave dynamics that have been studied for homogeneous flows in rotating systems that will have their counterparts for internal waves include the problem of nonlinear Rossby adjustment in a channel (Helfrich et al. 1999, Tomasson \& Melville 1992) and the related problem of Kelvin jumps (or shocks) in the coastal and equatorial waveguides (Fedorov \& Melville 1995, 1996, 2000).

\subsection{Dissipation}

The dissipation of waves as they propagate, and their final fate as they move into shallower waters, of interest for a variety of fundamental and practical reasons. If the length and timescales of decay are sufficiently short, then the asymptotic solutions of the simplest conservative models may not be observable nor relevant. 
If the final fate of the waves involves breaking and mixing in shallow water, then it may be of direct practical interest in modeling transport and mixing in the coastal ocean.

Nonlinear internal waves may be dissipated by boundary shear, interfacial shear, radiation damping, or localized breaking. The extent to which each contributes to the overall dissipation is not clear from observations, and isolating the contribution of vertical mixing caused by large internal waves in the coastal regions, when compared to other processes, may prove difficult.

3.3.1. Radiation damping. We have already mentioned radiation damping of nonlinear Kelvin waves by the generation of a wake of Poincaré waves. Nonlinear internal waves propagating along a shallow thermocline above a weakly stratified deep lower layer may radiate internal waves into the lower layer, thereby damping the nonlinear waves. This problem was formulated by Maslowe \& Redekopp (1980), who derived the inhomogeneous Benjamin-Ono equation and found adiabatic solutions for the decay rate of the solitary waves of the homogenous equation. Numerical solutions of the inhomogeneous evolution equation by Pereira \& Redekopp (1980) showed that the adiabatic solutions overestimated the damping, a result associated with the fact that the low (horizontal) wave numbers are damped more rapidly than the larger wave numbers.

3.3.2. Boundary shear. As described above, the need to directly consider the dissipative effects of boundary layers becomes apparent in comparing laboratory measurements and the simplest two-layer inviscid $\mathrm{KdV}$ models. In such cases the Reynolds numbers may be sufficiently small that viscous boundary-layer effects (including the interface) can be formulated to modify the evolution equation, with satisfactory results when compared with measurements (Helfrich \& Melville 1986). However, in the field, the flow will be turbulent, the bottom boundary will not normally be smooth, and resort must be made to parameterization of these effects in practical models. Holloway et al. (1997) provide a practical example of the use of an eKdV model with a quadratic bottom drag law with a Chézy coefficient to represent vertical turbulent transport of momentum and an eddy viscosity formulation for horizontal transport. They found it necessary to model the effects of boundary turbulence to achieve reasonable agreement with observations of the transformation of the internal tide over the northwest shelf of Australia (see also Vlasenko \& Hutter 2002).

3.3.3. Shear instability. Internal waves in a two-layer system separated by a thin stratified interface lead to a shear flow across the interface, which may become unstable when the inertial effects of the shear are not stabilized by the effects of gravity. For steady shear flows, appeal is usually made to Miles's theorem (see also Howard's semicircle theorem), which states that a necessary condition for instability is that the gradient Richardson number be somewhere less than $1 / 4$. However, on the basis of empirical evidence, this is often used as a sufficient condition for instability. This is the approach taken by Bogucki \& Garrett (1993) (c.f., Phillips 1977) for Boussinesq solitary waves, finding that if the wave amplitude $a \geq a_{c}=2\left(\delta b b_{1}\right)^{1 / 2}$, then the 


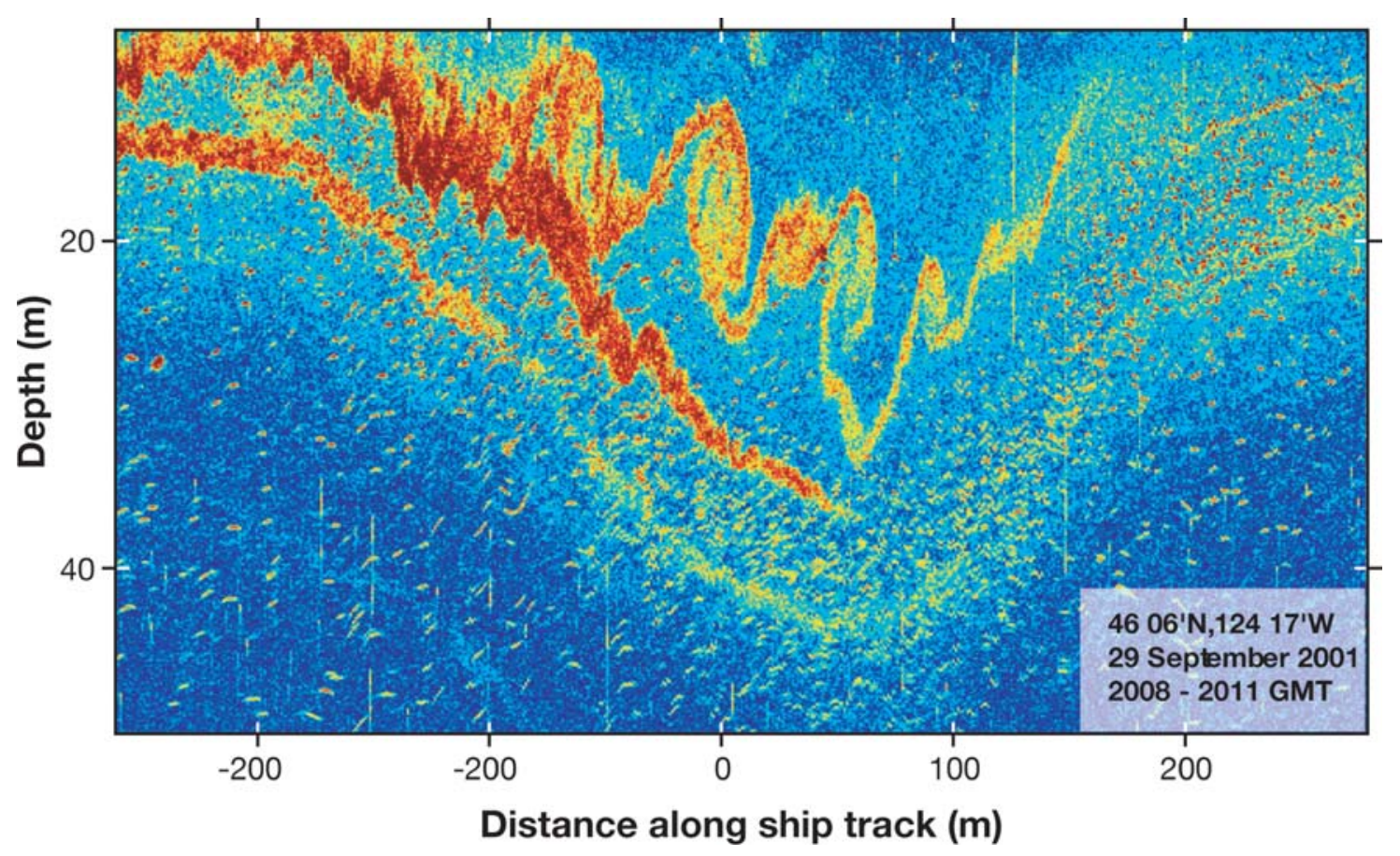

Figure 8

An acoustic backscatter record from Moum et al. (2003) showing Kelvin-Helmholtz billows growing from instabilities on the forward face of a solitary wave. The wave is propagating from left to right in the figure.

Richardson number $R i=\frac{g^{\prime} \delta b}{(\Delta u)^{2}} \leq \frac{1}{4}$, where $g^{\prime}$ is the reduced gravity, $\delta b$ is the thickness of the interface, and $\Delta u$ is the velocity jump across the interface, $\delta b \ll b_{1}, b_{1} \ll b_{2}$, and $a \ll b_{1}$. They assumed that instability leads to a thickening of the interface, with the required potential energy coming from the solitary wave, and used semiempirical stratified mixing models to predict the decay rate of the waves. The essential elements of their model appear to be consistent with the laboratory experiments of Grue et al. (1999).

Perhaps the best evidence for the incidence of Kelvin-Helmholtz instabilities in large internal waves comes from the recent field measurements of Moum et al. (2003). Figure 8, taken from their paper, shows acoustic imaging of Kelvin-Helmholtzlike billows growing from instabilities on the forward face of a strongly nonlinear internal wave. Here the billows grow to vertical scales of approximately $10 \mathrm{~m}$ and horizontal scales of approximately $50 \mathrm{~m}$. The authors comment that their observations appear to follow the descriptions by Grue et al. (2000) of similar instabilities in the laboratory, but both laboratory and field measurements do not have the resolution to unambiguously associate the onset of the instabilities with a fully resolved local Richardson number. 
3.3.4. Wave breaking. The essential features of kinematic wave breaking in hyperbolic systems are covered in Whitham (1974), where the onset of multivalued solutions, which occur on the leading face of the wave, are inferred to correspond to the formation of shocks or hydraulic jumps. Similar phenomena may occur for the evolution equations describing internal waves when the dispersive effects, which always suppress breaking, can be neglected compared to nonlinear effects. However, now breaking on the rear face of the wave may also occur and lead to the formation of dissipative internal hydraulic jumps. Smyth \& Holloway (1988) provide an extensive treatment of this class of problems in the context of observations of tidally generated internal waves and hydraulic jumps on the northwestern Australian shelf.

In laboratory experiments, Helfrich \& Melville (1986) found that breaking could occur in the neighborhood of the turning point, leading to a volume of mixed fluid that would evolve into a second-mode solitary-like wave propagating shoreward. In a laboratory study of the shoaling of waves of depression over a uniform slope, Helfrich (1992) found that breaking was followed by the formation of waves of elevation (or boluses) containing mixed fluid propagating up the slope, as seen by Wallace \& Wilkinson (1988) for periodic trains of long internal waves incident on a slope. Of the energy lost from internal waves, $15 \pm 5 \%$ (a measure of the "mixing efficiency") was expended in increasing the potential energy of the stratification. In a more extensive study of the shoaling of solitary waves, including large-amplitude waves, Michallet \& Ivey (1999) found that the breaking and mixing on the rear face of the incident wave appeared to follow separation of the offshore flow in the lower layer, consistent with the interpretation of Wallace \& Wilkinson (1988). They found that the mixing efficiency, which reached a maximum of $25 \%$ in their experiments, depended on the ratio of the characteristic horizontal scale of the incident wave to the scale of the slope.

The use of $\mathrm{KdV}$-type equations to model wave evolution to breaking is limited, and in recent years a number of fully numerical studies of the propagation of internal solitary waves over shoaling topography have been undertaken. Lamb $(2002,2003)$ considered the formation of waves over shoaling topography, and in the second paper explored the hypothesis that the configuration of the waves formed by shoaling may be related to the limiting form of the corresponding solitary waves. Specifically, if the limiting form of the waves includes a conjugate flow, then no trapped core is formed. However, if the limiting form corresponds to the maximum horizontal velocity in the wave matching the wave speed, then such a core may form (c.f., Grue et al. 2000).

Vlasenko \& Hutter (2002) solved the Reynolds-averaged equations, parameterized with eddy diffusivities, for the propagation of solitary waves of depression over slopeshelf topography for wave amplitudes and background stratifications comparable to those seen in the Andaman and Sulu seas. The solutions provide a detailed analysis of the evolution of the flow, which is notable for the development of a breaking criterion based on the initial wave amplitude and stratification and the slope of the bottom topography (c.f., Helfrich \& Melville 1986). Figure 9 from Vlasenko \& Hutter (2002) shows an example of wave evolution through the breaking point. Their 


\section{Distance (km)}
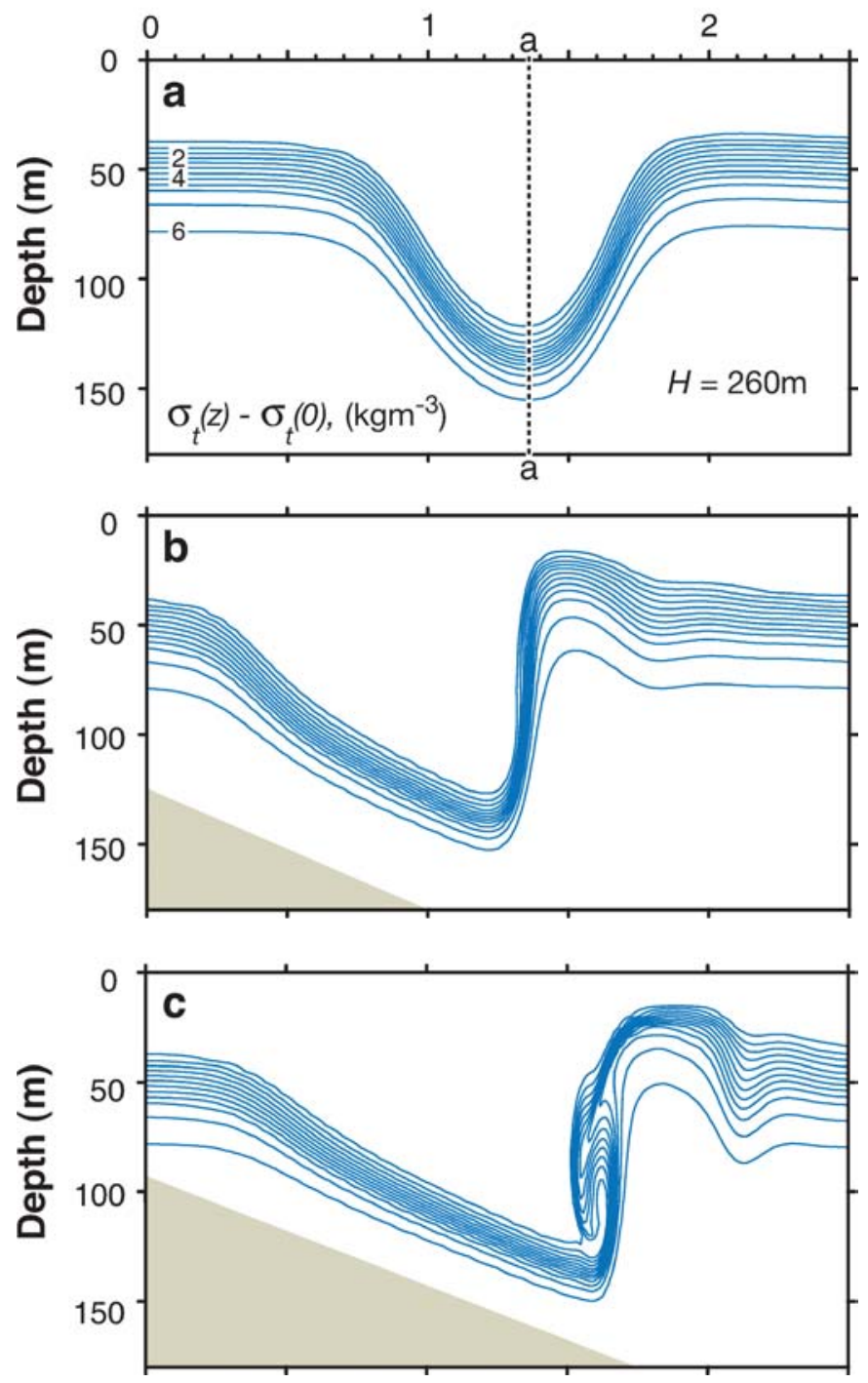

Figure 9

A numerical solution from Vlasenko \& Hutter (2002) showing the breaking of a large-amplitude solitary wave as it propagates over shoaling topography through a turning point. (a) The incident wave in deep water. (b) Incipient breaking $(c)$ just after the interface overturns.

calculations clearly show that the breaking is due primarily to kinematic overturning of the trailing face of the wave.

\section{DISCUSSION}

Developments in theoretical, laboratory, observational, and computational capabilities have provided the foundations for the progress of the past 40 years. Some advances have been striking, with measurements exemplified by Figure 8 showing that 
the ability to make detailed field measurements may now be comparable to that in the laboratory. The development of nested coupled ocean circulation models is approaching the time when long nonlinear internal wave dynamics and its influence on mixing and transport in the coastal oceans may need to be explicitly included to adequately resolve tidal forcing. For physical oceanographers, the so-called "tidal conversion" problem, the generation of the internal tides by the surface tides, remains an issue of great importance with implications for global tidal energy budgets, tidal drag on the Earth, and global budgets of ocean mixing (Munk \& Wunsch 1998, Rudnick et al. 2003).

An important class of questions concerns the processes that lead to the breaking or instability of long nonlinear internal waves and the subsequent evolution of the waves and turbulence. Underlying these dynamical questions is the issue of whether repeated wave-induced mixing events are significant contributors to the overall mixing in the coastal ocean, where other processes (e.g., surface buoyancy fluxes, mechanical mixing from the wind and Langmuir circulations, surface wave breaking) are important. Recent observations on the New England inner shelf by Pritchard \& Weller (2005) indicate the answer may be yes, at least during the summer when the winds are weaker. Obviously, more observational studies are necessary and, although nonlinear wave models are useful for investigating kinematic breaking, it is likely that numerical modeling, including large eddy simulation, will be required to investigate the subsequent wave-turbulence flows.

Our emphasis on low-mode waves is appropriate in light of the observational evidence, but it is also limiting, particularly in deeper water where the waveguide and forcing make a ray, or multimodal, description more appropriate. Low-mode wave packets have been observed in open ocean areas away from topography; for example, in the Bay of Biscay (New \& Da Silva 2002). Gerkema (2001) showed these waves could be generated by the scattering of an internal tidal beam on a near-surface pycnocline. By similar processes an internal tidal beam incident from the deep ocean could scatter into packets of low-mode internal waves on the continental shelf. This generation process has not been considered in any detail, but is likely a critical part of the evolution of the internal wave spectrum from deep to shallow water.

For the most part we have concentrated on almost unidirectional flows, but it is clear from related research in single-layer flows that three-dimensional effects can be important. SAR imagery of the coastal oceans typically shows that there are numerous sources of internal waves near the shelf break. The localized wave generation also leads to three-dimensional interacting wave fields on the shelf. For example, the SAR image of the Gulf of California (Figure 1) shows interacting wave fields in the northern reaches of the gulf. The image shows some evidence of Mach stems between oblique interacting waves. This is evidence of a three-wave resonance, which has been investigated for surface waves (Miles 1980) but not for internal waves. More generally, studies of full three-dimensional generation and interactions of long internal waves will, at a minimum, require formulations that are comparable to the Boussinesq equations for surface waves (Lynett \& Liu 2002, Tomasson \& Melville 1992), and full nonlinear numerical models to assess wave breaking. 


\section{ACKNOWLEDGMENTS}

We thank Tim Duda, Lee Fu, Ben Holt, Xiaofeng Li, Jim Moum, Steve Ramp, Tim Stanton, and Vasiliy Vlasenko for original figures from their publications. KRH acknowledges support from NSF and ONR and an Independent Study Award from the Woods Hole Oceanographic Institution. WKM acknowledges support from NSF and ONR, which has made his work in this area possible, in close collaboration with former graduate students at Scripps Institution of Oceanography and MIT. They include the senior author of this review, Peter Chang, Elizabeth Macomb, Gunnar Tomasson, and Alexey Fedorov. This is Woods Hole Oceanographic Institution Contribution number 11383.

\section{LITERATURE CITED}

Ablowitz MJ, Segur H. 1981. Solitons and the Inverse Scattering Transform. Philadelphia: SIAM. 425 pp.

Akylas TR. 1991. On the radiation damping of a solitary wave in a rotating channel. In Mathematical Approaches in Hydrodynamics, ed. T Miloh, pp. 175-81. Philadelphia: SIAM

Akylas TR. 1994. Three-dimensional long water-wave phenomena. Annu. Rev. Fluid Mech. 26:191-210

Apel JR Byrne, HM Proni JR, Charnell RL. 1975. Observations of oceanic internal and surface waves from the Earth resources technology satellite. 7. Geophys. Res. $80: 865-81$

Benjamin TB. 1966. Internal waves of finite amplitude and permanent form. F. Fluid Mech. 25:241-70

Benjamin TB. 1967. Internal waves of permanent form in fluids of great depth. 7. Fluid Mech. 29:559-92

Benney DJ. 1966. Long nonlinear waves in fluid flows. 7. Math. Phys. 45:52-63

Benney DJ, Ko DRS. 1978. The propagation of long, large-amplitude, internal waves. Stud. Appl. Math. 59:187-99

Bogucki D, Garrett C. 1993. A simple model for the shear-induced decay of an internal solitary wave. 7. Phys. Oceanogr. 23:1767-76

Brown DJ, Christie DR. 1998. Fully nonlinear solitary waves in continuously stratified incompressible Boussinesq fluids. Phys. Fluids 10:2569-86

Choi W, Camassa R. 1999. Fully nonlinear internal waves in a two-fluid system. 7. Fluid Mech. 396:1-36

Christie DR. 1992. The morning glory of the Gulf of Carpentaria: A paradigm for non-linear waves in the lower atmosphere. Aust. Meteorol. Mag. 41:21-60

Davis RE, Acrivos A. 1967. Solitary waves in deep water. 7. Fluid Mech. 29:593-601

Derzho OG, Grimshaw R. 1997. Solitary waves with a vortex core in a shallow layer of stratified fluid. Phys. Fluids 9:3378-85

Djordjevic VD, Redekopp LG. 1978. The fission and disintegration of internal solitary waves moving over two-dimensional topography. F. Phys. Oceanogr. 8:101624 
Duda TF, Lynch JF, Irish JD, Beardsley RC, SR Ramp, et al. 2004. Internal tide and nonlinear wave behavior in the continental slope in the northern South China Sea. IEEE 7. Ocean. Eng. 29:1105-31

Evans WA, Ford MJ. 1996. An integral equations approach to internal (2-layer) solitary waves. Phys. Fluids 8:2032-47

Farmer D, Armi L. 1999. The generation and trapping of solitary waves over topography. Science 283:188-90

Fedorov AV, Melville WK. 1995. Propagation and breaking of nonlinear Kelvin waves. F. Phys. Oceanogr. 25:2518-31

Fedorov AV, Melville WK. 1996. Hydraulic jumps at boundaries in rotating fluids. 7. Fluid Mech. 324:55-82

Fedorov AV, Melville WK. 2000. Kelvin fronts on the equatorial thermocline. F. Phys. Oceanogr: 30:1692-705

Fructus D, Grue J. 2004. Fully nonlinear solitary waves in a layered stratified fluid. 7. Fluid Mech. 505:323-47

Fu LL, Holt B. 1982. SEASAT views oceans and sea ice with synthetic aperture radar. NASA/JPL Publ. 81-120. Calif. Inst. Technol., Pasadena

Funakoshi M, Oikawa M. 1986. Long internal waves of large amplitude in a two-layer fluid. 7. Phys. Soc. Fpn. 55:128-44

Gardner CS, Greene JM, Kruskal MD, Muira RM. 1967. Method for solving the Korteweg-de Vries equation. Phys. Rev. Lett. 19:1095-97

Gerkema T. 1996. A unified model for the generation and fission of internal tides in a rotating ocean. 7. Mar. Res. 54:421-50

Gerkema T. 2001. Internal and interfacial tidal tides: beam scattering and local generation of solitary waves. F. Mar. Res. 59:227-55

Gerkema T, Zimmerman JTF. 1995. Generation of nonlinear internal tides and solitary waves. 7. Phys. Oceanogr. 25:1081-95

Grimshaw R. 1985. Evolution equations for weakly nonlinear, long internal waves in a rotating fluid. Stud. Appl. Math. 73:1-33

Grimshaw R. 1997. Internal solitary waves. In Advances in Coastal and Oceanographic Engineering, ed. P-LF Liu, pp. 1-30. Singapore: World Sci. 288 pp.

Grimshaw R, Melville WK. 1989. On the derivation of the modified KadomtsevPetviashvili equation. Stud. Appl. Math. 80:183-202

Grimshaw R, Pelinovsky E, Poloukhina O. 2002. Higher-order Korteweg-de Vries models for internal solitary waves in a stratified shear flow with a free surface. Nonlinear Proc. Geophys. 9:221-35

Grimshaw R, Pelinovsky E, Talipova T, Kurkin A. 2004. Simulation of the transformation of internal solitary waves on oceanic shelves. F. Phys. Oceanogr. 34:277491

Grimshaw R, Yi Z. 1991. Resonant generation of finite amplitude waves by the flow of a uniformly stratified fluid over topography. F. Fluid Mech. 229:603-28

Grimshaw RHJ. 1981. Evolution equations for long, nonlinear internal waves in stratified shear flows. Stud. Appl. Math. 65:159-88

Grimshaw RHJ, He J-M, Ostrovsky LA. 1997a. Terminal damping of a solitary wave due to radiation in rotational systems. Stud. Appl. Math. 101:197-210 
Grimshaw RHJ, Ostrovsky LA, Shrira VI, Stepanyants YA. 1998. Long nonlinear surface and internal gravity waves in a rotating ocean. Surv. Geophys. 19:289338

Grimshaw RHJ, Pelinovsky E, Talipova T. 1997b. The modified Korteweg-de Vries equation in the theory of large amplitude internal waves. Nonlinear Proc. Geophys. $4: 237-50$

Grimshaw RHJ, Smyth N. 1986. Resonant flow of a stratified fluid over topography. 7. Fluid Mech. 169:429-64

Grimshaw RHJ, Tang S. 1990. The rotation-modified Kadomtsev-Petviashvili equation: an analytrical and numerical study. Stud. Appl. Math. 83:223-48

Grue J, Friis HA, Palm E, Rusas P-O. 1997. A method for computing unsteady fully nonlinear interfacial waves. F. Fluid Mech. 351:223-52

Grue J, Jensen A, Rusas P-O, Sveen JK. 1999. Properties of large-amplitude internal waves. F. Fluid Mech. 380:257-78

Grue J, Jensen A, Rusas P-O, Sveen JK. 2000. Breaking and broadening of internal solitary waves. F. Fluid Mech. 413:181-217

Halpern D. 1971. Observations of short period internal waves in Massachusetts Bay. 7. Mar. Res. 29:116-32

Hammack JL, Segur H. 1978. Modelling criteria for long water waves. F. Fluid Mech. 84:359-73

Haury LR, Briscoe MG, Orr MH. 1979. Tidally generated internal wave packets in Massachusetts Bay. Nature 278:312-17

Helfrich KR. 1984. On long nonlinear internal waves over bottom topography. $\mathrm{PhD}$ thesis. MIT. 272 pp.

Helfrich KR. 1992. Internal solitary wave breaking and run-up on a uniform slope. 7. Fluid Mech. 243:133-54

Helfrich KR, Kuo AC, Pratt LJ. 1999. Nonlinear Rossby adjustment in a channel. $\mathcal{F}$. Fluid Mech. 390:187-222

Helfrich KR, Melville WK. 1986. On long nonlinear internal waves over slope-shelf topography. 7. Fluid Mech. 167:285-308

Helfrich KR, Melville WK, Miles JW. 1984. On interfacial solitary waves over slowly varying topography. F. Fluid Mech. 149:305-17

Holloway PE, Pelinovsky E, Talipova T. 1999. A generalized Korteweg-de Vries model of internal tide transformation in the coastal zone. 7. Geophys. Res. 104(C8):18333-50

Holloway PE, Pelinovsky E, Talipova T, Barnes B. 1997. A nonlinear model of internal tide transformation on the Australian north west shelf. 7 . Phys. Oceanogr. 27:871-96

Hunkins K, Fliegel M. 1973. Internal undular surges in Seneca Lake: a natural occurence of solitons. 7. Geophys. Res. 78:539-48

Jo T-C, Choi W. 2002. Dynamics of strongly nonlinear internal solitary waves in shallow water. Stud. Appl. Math. 109:205-27

Joseph RI. 1977. Solitary waves in finite depth fluids. F. Phys. A 10:1225-27

Kadomtsev BB, Petviashvili VI. 1970. On the stability of solitary waves in weakly dispersing media. Sov. Phys. Dokl. 15:539-41 
Kakutani T, Matsuuchi K. 1975. Effect of viscosity on long gravity waves. F. Phys. Soc. 7pn. 39:237-46

Kakutani T, Yamasaki N. 1978. Solitary waves on a two-layer fluid. F. Phys. Soc. Fpn. 45:674-79

Katsis C, Akylas TR. 1987. Solitary internal waves in a rotating channel: a numerical study. Phys. Fluids 30:297-301

Klymak JM, Moum JN. 2003. Internal solitary waves of elevation advancing on a shoaling shelf. Geophys. Res. Lett. 30:2045

Knickerbocker CJ, Newell AC. 1980. Internal solitary waves near a turning point. Phys. Lett. A 75:326-30

Koop CG, Butler C. 1981. An investigation of internal solitary waves in a two-fluid system. F. Fluid Mech. 112:225-51

Kubota T, Ko DRS, Dobbs LD. 1978. Weakly-nonlinear long internal waves in a stratified fluid of finite depth. F. Hydronaut. 12:157-65

Lamb KG. 2002. A numerical investigation of solitary internal waves with trapped cores formed via shoaling. F. Fluid Mech. 451:109-44

Lamb KG. 2003. Shoaling solitary internal waves: on a criterion for the formation of waves with trapped cores. F. Fluid Mech. 478:81-100

Lamb KG. 2004. On boundary-layer separation and internal wave generation at the Knight Inlet sill. Proc. R. Soc. London Ser. A 460:2305-37

Lamb KG, Wilkie KP. 2004. Conjugate flows for waves with trapped cores. Phys. Fluids 16:4685-95

Lamb KG, Yan L. 1996. The evolution of internal wave undular bores: comparison of a fully-nonlinear numerical model with weakly nonlinear theories. 7 . Phys. Ocean. 26:2712-34

Lee C-Y, Beardsley RC. 1974. The generation of long nonlinear internal waves in a weakly stratified shear flow. F. Geophys. Res. 79:453-62

Leonov AI. 1981. The effect of the earth's rotation on the propagation of weak nonlinear surface and internal long oceanic waves. Ann. NY Acad. Sci. 373:15059

Li XF, Dong CM, Clemente-Colon P, Pichel WG, Friedman KS. 2004. Synthetic aperture radar observation of the sea surface imprints of upstream atmospheric solitons generated by flow impeded by an island. 7. Geophys. Res. 109(C2):CO2016

Liu AK, Chang YS, Hsu M-K, Liang NK. 1998. Evolution of nonlinear internal waves in the East and South China Seas. 7. Geophys. Res. 103(C4):7995-8008

Long RR. 1953. Some aspects of the flow of stratified fluids. I. A theoretical investigation. Tellus 42:42-58

Long RR. 1956. Solitary waves in one- and two-fluid systems. Tellus 8:460-71

Lynch JF, Ramp SR, Chin C-S, Tang TY, Yang Y-J, Simmen JA. 2004. Research highlights from the Asian Seas International Acoustics Experiment in the South China Sea. IEEE 7. Ocean. Eng. 29:1067-74

Lynett PJ, Liu PL-F. 2002. A two-dimensional, depth-integrated model for internal wave propagation over variable bathymetry. Wave Motion 36:221-40

Maslowe SA, Redekopp LG. 1980. Long nonlinear waves in stratified shear flows. 7. Fluid Mech. 101:321-48 
Matsuura T, Hibiya T. 1990. An experimental and numerical study of the internal wave generation by tide-topography interaction. F. Phys. Oceanogr. 20:506-21

Maxworthy T. 1979. A note on the internal solitary waves produced by tidal flow over a three-dimensional ridge. 7. Geophys. Res. 84:338-46

Maxworthy T. 1980. On the formation of nonlinear internal waves from the gravitational collapse of mixed regions in two and three dimensions. F. Fluid Mech. 896:47-64

Maxworthy T. 1983. Experiments on solitary internal Kelvin waves. 7. Fluid Mech. $129: 365-83$

Melville WK, Helfrich KR. 1987. Transcritical two-layer flow over topography. 7 . Fluid Mech. 178:31-52

Melville WK, Tomasson GG, Renouard DP. 1989. On the stability of Kelvin waves. 7. Fluid Mech. 206:1-23

Michallet H, Barthelemy E. 1998. Experimental study of interfacial solitary waves. 7. Fluid Mech. 366:159-77

Michallet H, Ivey GN. 1999. Experiments on mixing due to internal solitary waves breaking on uniform slopes. 7. Geophys. Res. 104(C6):13467-78

Miles JW. 1976. Korteweg-de Vries equation modified by viscosity. Phys. Fluids 19:1063

Miles JW. 1979. On internal solitary waves. Tellus 31:456-62

Miles JW. 1980. Solitary waves. Annu. Rev. Fluid Mech. 12:11-43

Miyata M. 1985. An internal solitary wave of large amplitude. La Mer 23:43-48

Miyata M. 1988. Long internal waves of large amplitude. In Nonlinear Water Waves, IUTAM Symp., Tokyo 1987, ed. K Horikawa, H Maruo, pp. 399-406. Berlin: Springer-Verlag. 466 pp.

Moum JN, Farmer DM, Smyth WD, Armi L, Vagle S. 2003. Structure and generation of turbulence at interfaces strained by internal solitary waves propagating shoreward over the continental shelf. F. Phys. Oceanogr. 33:2093-112

Munk W, Wunsch C. 1998. Abyssal recipes II: energetics of tidal and wind mixing Deep Sea Res. I 45:1977-2010

New AL, Da Silva JCB. 2002. Remote sensing evidence for the local generation of internal soliton packets in the Central Bay of Biscay. Deep Sea Res. I 49:91534

Odolu AB. 1978. On the equations of long nonlinear waves in the ocean. Okeanologiia 18:965-71 (In Russian)

Ono H. 1975. Algebraic solitary waves in stratified fluids. 7. Phys. Soc. Fpn. 39:1082-91

Orr MH, Mignerey PC. 2003. Nonlinear internal waves in the South China Sea: observation of the conversion of depression internal waves to elevation internal waves. F. Geophys. Res. 108(C3):3064

Osborne AR, Burch TL. 1980. Internal solitons in the Andaman Sea. Science 208:45160

Ostrovsky L. 1978. Nonlinear internal waves in a rotating ocean. Oceanogology 18(2):119-25

Ostrovsky LA, Grue J. 2003. Evolution equations for strongly nonlinear internal waves. Phys. Fluids 15:2934-48 
Ostrovsky LA, Stepanyants YA. 1989. Do internal solitons exist in the ocean? Rev. Geophys. 27:293-310

Pereira NR, Redekopp LG. 1980. Radiation damping of long, finite-amplitude internal waves. Phys. Fluids 23:2182-83

Perry RB, Schimke GR. 1965. Large amplitude internal waves observed off the northwest coast of Sumatra. 7. Geophys. Res. 70:2319-24

Phillips OM. 1977. The Dynamics of the Upper Ocean. Cambridge, UK: Cambridge Univ. Press. 336 pp.

Pritchard M, Weller RA. 2005. Observations of internal bores and waves of elevation on the New England inner continental shelf during summer 2001. F. Geophys. Res. 110(C3):C03020

Pullin DI, Grimshaw RHJ. 1988. Finite amplitude solitary waves on the interface between two fluids. Phys. Fluids 31:3350-59

Ramp SR, Tang TY, Duda TF, Lynch JF, Liu AK, et al. 2004. Internal solitons in the northeastern South China Sea Part I: sources and deep water propagation. IEEE 7. Ocean. Eng. 29:1157-81

Renouard DP, Chabert d'Hières G, Zhang X. 1987. An experimental study of strongly nonlinear waves in a rotating system. F. Fluid Mech. 177:381-94

Rudnick DL, Boyd TJ, Brainard RE, Carter GS, Egbert GD, et al. 2003. From tides to mixing along the Hawaiian ridge. Science 301:355-57

Segur H, Hammack JL. 1982. Soliton models of long internal waves. F. Fluid Mech. 118:285-304

Smyth N, Holloway P. 1988. Hydraulic jump and undular bore formation on a shelf break. F. Phys. Oceanogr. 18:947-62

Stamp AP, Jacka M. 1996. Deep-water internal solitary waves. F. Fluid Mech. 305:34771

Stanton TP, Ostrovsky LA. 1998. Observations of highly nonlinear solitons over the continental shelf. Geophys. Res. Lett. 25:2695-98

Stastna M, Lamb KG. 2002. Large fully nonlinear internal solitary waves: the effect of background current. Phys. Fluids 14(9):2987-99

Stastna M, Peltier WR. 2004. Upstream-propagating solitary waves and forced internal-wave breaking in a stratified flow over a sill. Proc. R. Soc. London Ser. A 460:3159-90

Thorpe SA. 1971. Asymmetry of the internal wave seiche in Loch Ness. Nature 231:306-8

Tomasson GG, Melville WK. 1992. Geostrophic adjustment in a channel: nonlinear and dispersive effects. F. Fluid Mech. 241:23-48

Tung KK, Chan TF, Kubota T. 1982. Large amplitude internal waves of permanent form. Stud. Appl. Math. 66:1-44

Turkington B, Eydeland A, Wang S. 1991. A computational method for solitary waves in a continuously stratified fluid. Stud. Appl. Math. 85:93-127

Turner REL, Vanden-Broeck J-M. 1988. Broadening of interfacial solitary waves. Phys. Fluids 31:2486-90

Vlasenko V, Hutter K. 2002. Numerical experiments on the breaking of solitary internal waves over a slope-shelf topography. 7. Phys. Oceanogr. 32(6):177993 
Vlasenko VI, Hutter K. 2001. Generation of second mode solitary waves by the interaction of a first mode soliton with a sill. Nonlinear Proc. Geophys. 8:22339

Wallace BC, Wilkinson DL. 1988. Run-up of internal waves on a gentle slope in a two-layered system. F. Fluid Mech. 191:419-42

Whitham GB. 1974. Linear and Nonlinear Waves. New York: Wiley. 636 pp.

Ziegenbein J. 1969. Short internal waves in the Strait of Gibraltar. Deep Sea Res. $16: 479-87$

Ziegenbein J. 1970. Spatial observations of short internal waves in the Strait of Gibraltar. Deep Sea Res. 17:867-75 


\section{Contents}

Annual Review of

Fluid Mechanics

Volume 38, 2006

Nonlinear and Wave Theory Contributions of T. Brooke Benjamin

(1929-1995)

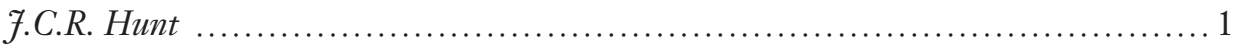

Aerodynamics of Race Cars

Foseph Katz

Experimental Fluid Mechanics of Pulsatile Artificial Blood Pumps

Steven Deutsch, Fobn M. Tarbell, Keefe B. Manning, Gerson Rosenberg,

and Arnold A. Fontaine

Fluid Mechanics and Homeland Security

Gary S. Settles

Scaling: Wind Tunnel to Flight

Dennis M. Bushnell

Critical Hypersonic Aerothermodynamic Phenomena

John 7. Bertin and Russell M. Cummings

Drop Impact Dynamics: Splashing, Spreading, Receding, Bouncing...

A.L. Yarin

Passive and Active Flow Control by Swimming Fishes and Mammals F.E. Fish and G.V. Lauder

Fluid Mechanical Aspects of the Gas-Lift Technique

S. Guet and G. Ooms

Dynamics and Control of High-Reynolds-Number Flow over Open

Cavities

Clarence W. Rowley and David R. Williams .....

Modeling Shapes and Dynamics of Confined Bubbles

Vladimir S. Ajaev and G.M. Homsy

Electrokinetic Flow and Dispersion in Capillary Electrophoresis

Sandip Ghosal.

Walking on Water: Biolocomotion at the Interface

Fobn W.M. Bush and David L. Hu 
Biofluidmechanics of Reproduction

Lisa 7. Fauci and Robert Dillon

Long Nonlinear Internal Waves

Karl R. Helfrich and W. Kendall Melville 395

Premelting Dynamics

7.S. Wettlaufer and M. Grae Worster

Large-Eddy Simulation of Turbulent Combustion

Heinz Pitsch 453

Computational Prediction of Flow-Generated Sound

Meng Wang, Fonathan B. Freund, and Sanjiva K. Lele 483

\section{INDEXES}

Subject Index 513

Cumulative Index of Contributing Authors, Volumes 1-38

Cumulative Index of Chapter Titles, Volumes 1-38

\section{ERRATA}

An online log of corrections to Annual Review of Fluid Mechanics chapters may be found at http://fluid.annualreviews.org/errata.shtml 